\title{
Effectiveness of a theory-informed documentary to reduce consumption of meat and animal products: Three randomized controlled experiments
}

Maya B. Mathur*1, Jacob R. Peacock ${ }^{\dagger 2}$, Thomas N. Robinson ${ }^{\ddagger 3}$, and Christopher D. Gardner ${ }^{\S 4}$

${ }^{1}$ Department of Pediatrics and Quantitative Sciences Unit, Stanford University

${ }^{2}$ The Humane League Labs

${ }^{3}$ Stanford Solutions Science Lab, Department of Pediatrics, Stanford University ${ }^{4}$ Stanford Prevention Research Center, Stanford University

- Citation: Mathur MB, Peacock JR, Robinson TN, \& Gardner CD (in press). Effectiveness of a theory-informed documentary to reduce consumption of meat and animal products: Three randomized controlled experiments. Nutrients. Preprint available at https://osf.io/vgu6z

- Word count: 8,683

- Keywords: meat consumption, dietary change, sustainability, education, behavior interventions

- Running head: "Documentary to reduce MAP consumption"

${ }^{*}$ Correspondence to: Maya B. Mathur (mmathur@stanford.edu), Department of Pediatrics and Quantitative Sciences Unit, 1701 Page Mill Road, Palo Alto, CA, 94304.

$\dagger$ jacob@rethinkpriorities.org

¥tom.robinson@stanford.edu

§cgardner@stanford.edu 


\begin{abstract}
(200/200)
Several societal issues could be mitigated by reducing global consumption of meat and animal products (MAP). In three randomized, controlled experiments ( $n=217$ to 574 ), we evaluated the effects of a documentary that presents health, environmental, and animal welfare motivations for reducing participants' reported MAP consumption. Study 1 assessed the documentary's effectiveness after 12 days using methodological innovations designed to minimize social desirability bias, a widespread limitation of past research. Study 2 investigated discrepancies between the results of Study 1 and the results of previous studies by further examining the role of social desirability bias. Study 3 assessed the documentary's effectiveness in a new population anticipated to be more responsive and upon enhancing the intervention content. We found that the documentary did not decrease MAP consumption when potential social desirability bias was minimized (Studies 1 and 3). The documentary also did not affect reported consumption among participants whose demographics suggested they might be more receptive (Study 3). However, the documentary did substantially increase intentions to reduce consumption, consistent with past studies (Studies 2 and 3). Overall, we conclude that some past studies of similar interventions may have overestimated effects due to methodological biases. Novel intervention strategies to reduce MAP consumption may be needed.
\end{abstract}




\section{INTRODUCTION}

Several exigent societal issues could be mitigated by reducing global consumption of meat and animal products (MAP) and encouraging predominantly plant-based diets in their place. Authoritative enjoinments for such a dietary shift have highlighted its potential to improve public health [1]6], reduce risks of zoonotic pandemics and antibiotic resistance [7], curb environmental degradation and climate change [3-6, 8], and limit the preventable suffering and slaughter of approximately 500 to 12,000 animals over the lifetime of each human consuming a diet typical of their country [6, 9]. Nevertheless, MAP consumption in Western countries far exceeds nutritional recommendations [3, 6] and, worldwide, continues to rise substantially [4]. Whereas existing research attention has often focused specifically on reducing consumption of red and processed meat [10, in this paper, we focus more broadly on shifting consumption of all meats (i.e., edible animal flesh) and animal products (i.e., eggs and dairy) to plant-based diets. Shifting consumption from red and processed meat to poultry, fish, dairy, and eggs would be beneficial for individual health, but probably less so than making comparable shifts to healthy plant-based foods (such as vegetables, fruits, whole grains, and legumes) that are severely lacking in the standard Western diet [3, 11, 12. Furthermore, producing poultry, fish, dairy, and eggs causes considerable environmental and ecological damage [13-15] and has severe animal welfare impacts [6].

Developing simple interventions to encourage dietary shifts from MAP to healthy plantbased foods could therefore carry widespread societal benefits. Educational interventions that make appeals to individual health [10, 16], the environment [10, 16], or animal welfare [16, 17] may be effective. More subtle "nudge" interventions that may operate outside participants' conscious awareness, for example by repositioning meat dishes to be less prominent in cafeterias, may also be effective [16, 18]. Although these types of interventions are promising, many existing studies have methodological limitations [17]. These include the potential for social desirability bias that could artificially inflate apparent intervention effects [19], 
measurement of outcomes only in terms of participants' attitudes or intended behavior rather than actual MAP consumption, and small sample sizes. As a result, we are aware of very few specific interventions that are adequately well-evidenced to strongly support their widespread dissemination at this point.

We conducted a series of parallel-group, randomized controlled experiments designed to help resolve these methodological challenges of previous studies. Namely, our studies took stringent precautions against social desirability bias, using longitudinal designs, and measured food consumption outcomes using food frequency questionnaires. The intervention was a 20-minute documentary that encourages dietary shifts from all meats and animal products to plant-based diets. We selected this documentary because its content reflects certain best practices for designing effective interventions in general, and its content also harnesses the specific psychology of MAP consumption [17]. In general, providing educational information can influence beliefs and intentions that may subsequently shape behavior [20]. The public appears to be poorly informed about the aforementioned consequences of global MAP consumption; in fact, many individuals appear to deliberately avoid such information [21]. Thus, providing information that helps remedy this knowledge gap may be effective. Additionally, portraying a desired behavior as aligning with social norms (what others believe one should do, or what others actually do) can effectively shift behaviors, including food choices [22, 23]. Providing concrete suggestions for how to change one's behavior (e.g., recipes) may help individuals to form concrete implementation intentions for what they plan to do when faced with food choices [24]. According to the Theory of Planned Behavior, providing simple suggestions may increase individuals' perceived ability to control their future behavior and their intentions to do so [20]. Indeed, previous interventions to reduce consumption of meat and/or animal products that invoked these components have obtained preliminarily promising results. Such interventions have included, for example, providing leaflets, news articles, and videos [17, 25-27].

In addition to leveraging these general components of effective behavioral interventions, the 
documentary we studied was designed to also harness the unique social, moral, and affective psychology underlying MAP consumption [28, 29]. For example, although ethical concern about factory farming conditions is now a majority stance in several developed countries [30], MAP consumption remains nearly universal. This discrepancy between people's ethical views and their actual behavior, termed the "meat paradox" [31, can induce cognitive dissonance. Previous interventions have successfully invoked this dissonance by using meat-animal reminders, which are simple visual or verbal reminders of the connection between MAP and animals (e.g., photographs of meat dishes presented next to photographs of the animals from which they came) [32 37]. Last, physical disgust and moral disgust are closely intertwined and powerfully shape food choices [38, 39]. Experiencing physical disgust can amplify negative moral judgments, and conversely, experiencing moral disgust can induce physical disgust [40]. Previous interventions to reduce consumption of meat and/or animal products have often invoked disgust by describing, for example, "crowded conditions [and] pens covered in excrement and germs" [41]. We also selected this documentary because it has been disseminated in practice via social media advertising by a nonprofit (The Humane League). For example, in 2019, the nonprofit's advertising generated 13 million visits to websites deploying documentary-driven interventions, including this documentary, resulting in 8 million minutes of viewing.

In Study 1, we aimed to assess the documentary's effectiveness using a study design that improved upon certain methodological limitations of previous work, described above. In Study 2, we aimed to adjudicate discrepancies between the results of Study 1 and the results of previous studies by further examining the role of social desirability bias. In Study 3, we aimed to assess the documentary's effectiveness in a different population and upon adding new components to the intervention, which were designed to increase participant engagement. To this end, in Studies 1 and 3, our primary outcome was participants' total MAP consumption over the past week (henceforth "consumption"), reported approximately 2 weeks after random assignment and exposure to the documentary. In both studies, we 
secondarily assessed consumption of specific categories of MAP as well as consumption of healthy plant-based foods. We also assessed the extent to which the intervention's effects might differ by participants' demographic characteristics; such findings could be used to cost-effectively target dissemination. For example, previous work has suggested that sex, education, and political liberalism could moderate the effectiveness of interventions to reduce consumption of meat and/or animal products [42-45]. Studies 1 and 3 took stringent precautions against social desirability bias. In Study 2, to further examine the potential for social desirability bias, our primary outcome was participants' immediate intentions to increase, decrease, or not change their consumption, similar to many existing studies in the literature.

\subsection{Methods}

For all 3 studies, we preregistered in detail all methods and statistical analyses, and the datasets and materials are publicly available (Section 7). All studies were approved by the Stanford University IRB (protocol \#57476). Statistical methods are detailed further in the Supplement. We conducted all statistical analyses in $\mathrm{R}$ [46], version 4.0.2.

\subsubsection{Study design, participants, and sample size determination}

We conducted a 2-arm, parallel-group, 12-day randomized controlled experiment comparing the documentary to an unrelated video, whose contents are detailed below. We conducted all studies online by embedding the videos in a questionnaire that we created in Qualtrics [47]. We used the online platform Prolific Academic to recruit United States-based participants who were at least 18 years old, without further demographic restrictions [48]. Prolific is a data-collection platform in which users can complete paid online research studies; the platform functions similarly to Amazon Mechanical Turk, but with apparently higher data quality [49]. 
Participants recruited through Prolific and similar platforms may be more demographically diverse than traditional undergraduate samples, but are typically not a representative sample of the United States 4.9 .

To maximize external generalizability and minimize social desirability bias, we used vague recruitment text that did not refer to MAP consumption or to motivations for reducing consumption [50]. We recruited 650 participants ${ }^{a}$ at the baseline wave of data collection $\left(T_{0}\right) I^{b}$ To minimize social desirability bias by blinding participants to the study's purpose, we used new recruitment text that described the $T_{1}$ wave as if it were a standalone study and did not reveal its connection to the $T_{0}$ wave. We refer to this approach as "naïve re-recruitment". At $T_{1}$, participants self-reported their consumption frequencies and typical serving sizes of 6 individual categories of meats (chicken, turkey, fish, pork, beef, other meat), 2 categories of animal products (dairy, eggs), 5 categories of healthy plant-based foods (leafy green vegetables, other vegetables, fruits, whole grains, legumes), and 2 decoy foods that were not analyzed (refined grains and sweetened beverages). We included the decoy foods to further conceal the purpose of the study. Participants also completed items to assess their awareness of the purpose of the study and exploratory attitude measures, detailed below All questionnaire items appear in the Supplement.

${ }^{a}$ We chose this sample size by assuming $85 \%$ retention such that we would analyze approximately 552 participants, yielding $80 \%$ power to detect an effect size of $d=0.24$ on the standardized mean difference scale, $95 \%$ power to detect an effect size of $d=0.31$, and an estimated confidence interval width of $d=0.33$. Secondarily, this sample size yields approximately $80 \%$ power to detect an interaction of the intervention with a balanced binary covariate of $d=0.34$.

${ }^{b}$ Individual follow-up times varied from 12 days to 19 days because participants did not necessarily have to complete the $T_{1}$ questionnaire the day we made it available. In order to achieve a typical follow-up time of approximately 2 weeks, we made the follow-up questionnaire available on Prolific 12 days after the date on which we closed baseline data collection, and it remained open for 7 days.

${ }^{c}$ The attitude measures appeared after the food consumption items and awareness probes because we expected that the attitude measures themselves could increase participants' awareness of the purpose of the study. 


\subsubsection{Intervention documentary and control video}

The intervention was a 20-minute documentary, Good For Us, produced by The Humane League ${ }^{d}$ and designed with close attention to psychological theory [51]. The documentary encourages plant-based diets that reduce consumption of all meats and animal products, thus differing from many existing interventions that focus on reducing consumption of red and processed meat in favor of not only plant-based foods but also poultry, fish, dairy, and eggs [10]. This broader focus aligns with the holistic societal concerns that motivated this research, as described in the Introduction.

The documentary was designed to shift explicit attitudes and intended behaviors [20] using educational appeals to individual health, to the environment and climate change, and to animal welfare. Through its multiple narrators, the documentary makes recommendations to eat, for example, "plant-based" and "vegetarian, and better yet, vegan" diets. In addition to these direct educational appeals, the documentary uses indirect means to shift dietary behavior, as discussed in the Introduction. First, the documentary invokes physical disgust by showing graphic footage of factory farms and slaughterhouses (e.g., of laying hens in filthy battery cages). Second, the documentary uses both verbale and visual meat-animal reminders (e.g., by cutting directly from footage of broiler chickens in a factory farm to footage of packaged chicken in a supermarket). Third, the documentary invokes social norms regarding increased demand for plant-based meals [] As described in the Introduction, psychological theory and empirical findings suggest that these elements may be potent means of reducing MAP consumption [17]. We added to the end of the documentary a brief screen stating "For practical tips on shifting to plant-based eating, see: www.eatingveg.org/how and www. eatingveg.org/what," These websites provide, for example, recipes and tips for

\footnotetext{
${ }^{d}$ We obtained permission from The Humane League for use of the documentary in the study.

${ }^{e}$ For example, a narrator states: "I feel like people eat animals because the process is so removed from us... When I used to eat meat, I didn't really connect that [the meat on my plate] used to be a live animal."

${ }^{f}$ For example, a narrator states: "Restaurants are catering to the millenial population that is really demanding...a vegan diet, a vegetarian diet, a flexitarian diet."
} 
handling social pressure from family and friends. Providing such practical tips may help individuals to form concrete implementation intentions, as discussed in the Introduction.

For the control group, we opted to present a control video rather than no video in order to match the duration of study between intervention and control participants, thus reducing the possibility of differential dropout between these groups. The control video was a 20 -minute TED talk by Brené Brown entitled "Listening to shame", which was a generic motivational speech that encouraged listeners to embrace the experience of feeling vulnerable. The content was unrelated to food choices and to any of the aforementioned reasons for reducing MAP consumption. We chose this video because its length matched that of the documentary and because pilot studies indicated that, like the documentary, participants found it engaging.

\subsubsection{Outcomes}

Participants reported their past week's consumption of each of the 6 categories of meats, 2 categories of animal products, 5 categories of healthy plant foods, and 2 decoy foods using a modified version of the National Cancer Institute's Dietary Health Questionnaire III (DHQ III). This scale was designed to reduce measurement error and has been validated through comparison to 24-hour food recalls [52 -54$] .7$ For each food category, participants answered the question "Over the past week, how often did you eat [food category]?" using 6 ordinal responses ranging from "never" to "2 or more times per day". Participants also reported the weights of foods consumed by answering the question "Each time you ate [food category], how much did you usually eat?" using 3 ordinal responses ("less than 2 ounces or less than $1 / 2$ cup", " 2 to 5 ounces or $1 / 2$ to 1 cup", or "more than 5 ounces or more than 1 cup").

${ }^{g}$ These websites have seen been moved and modified. Examples of content similar to what participants would have seen at www. eatingveg.org/how and www. eatingveg.org/what are publicly available (https: //osf.io/xrckh/).

${ }^{n}$ Validation studies comparing versions of the DHQ to 24-hour recalls estimated that these two measures had deattenuated correlations of approximately 0.50 for meats and eggs, and approximately 0.75 for dairy [54. We modified the 1-month DHQ-III that includes portion sizes. Our modifications were: (1) adapting the frequency options to correspond to our briefer follow-up time frame; (2) condensing individual foods into fewer categories, informed by those used in 26] to reduce survey fatigue; and (3) omitting a large number of foods not relevant to the present research. 
We estimated the total amount of each food category consumed by each participant by multiplying consumption frequencies by weights. Participants' total weight of MAP consumed over the past week was the primary food outcome, and their consumption of the individual foods were secondary outcomes.

We also collected exploratory measures of participants' attitudes and values regarding health, the environment, and animal welfare [55]. We also probed in more detail participants' attitudes regarding animal welfare because this type of educational appeal has appeared only relatively recently in dietary interventions [17]. These exploratory measures are detailed in the Supplement.

\subsubsection{Other measures}

At $T_{0}$, participants provided their sex, age, education level, race/ethnicity, current state and county of residence, and political party affiliation. We calculated an index of "county liberalism", representing the proportion of Democratic votes in the participant's county (Supplement). These measures were collected as possible moderators of intervention effects and for use in multiple imputation (Section 2.1.5). We did not measure consumption at baseline because doing so could have increased participants' awareness of the purpose of the study. Randomization ensures balance between groups, on average, on baseline consumption even though we did not measure this variable.

As an attention check, we asked participants, "Which of the following points did the video make? Please select all that apply." Participants could select any number of statements from among five. Exactly one statement was correct for the intervention group ("The ways we raise animals for human consumption causes the animals to suffer"), but was incorrect for the control group. The remaining 4 statements were plausible, but were incorrect for both groups (e.g., "Most Americans get less than the recommended amount of exercise"). Thus, if participants were perfectly attentive, all intervention participants would choose exactly one answer, while all control participants would not indicate any of the answers. 


\footnotetext{
${ }^{i}$ Harmonic mean $p$-values aggregate potentially non-independent $p$-values and represent an outcome-wide metric of the intervention's effect on the secondary outcomes [60].
} awareness of the study's purpose using 2 multiple-choice items that mimicked a funnel debriefing (Supplement). We coded participants as "potentially aware" if their responses indicated that they correctly believed that the researchers intended to decrease MAP consumption. Additionally, because we conducted this study during the SARS-CoV-2 pandemic, we asked participants to what extent the pandemic was affecting their ability to choose what they eat; this item was simply descriptive.

\subsubsection{Statistical analyses}

Analyses of primary and secondary outcomes We estimated the difference in mean total consumption between the intervention and control group and conducted a 2-sample Welch's $t$-test, which accommodates the heteroskedasticity and skewed errors that are typical of food outcome measures [56, 57]. We repeated this analysis for the secondary consumption outcomes and exploratory attitude outcomes. We calculated $p$-values for all secondary outcomes both with and without Bonferroni correction, counting one test per secondary outcome (corrected $\alpha=0.05 / 17$ tests $=0.0029)$ [58]. We tested the global null hypothesis that the intervention affected none of the secondary outcomes by calculating harmonic mean $p$-values [59] for all secondary outcomes considered together, for all secondary food outcomes considered together, and for all exploratory attitude outcomes considered together We also calculated the number of secondary outcomes with a Bonferroni-corrected $p<0.05$, which can be interpreted with $95 \%$ confidence as the number of secondary outcomes on which the intervention has a nonzero effect [61]. Throughout these analyses, we imputed missing data that arose from attrition at $T_{1}[\underline{62}, 63]$.

Analysis of moderators We examined 2-way interactions of the intervention with baseline participant characteristics: being female, being $\leq 25$ years old, having at least graduated

At $T_{1}$, for use in sensitivity analyses (Section 2.1.5), we assessed participants' potential 
from a 2-year college, being a Democrat (vs. being a Republican), being an Independent or reporting no party affiliation (vs. being a Republican), being Caucasian, $]$ and county liberalism (rescaled to represent a 10-percentage point higher share of Democratic votes in the participant's county). We included all of these candidate moderators simultaneously in a generalized least-squares model with heteroskedasticity-consistent robust standard errors [56]. We again reported inference both with and without Bonferroni correction, counting one test per moderator regression coefficient.

Sensitivity analyses We conducted 3 sensitivity analyses for the primary results. First, we conducted a complete-case analysis as a counterpart to the primary multiple imputation analyses. Second, anticipating that there may be more nondifferential measurement error (i.e., random noise) in participants' reporting of serving size volumes than in their reporting of consumption frequencies (e.g., because participants may have difficulty estimating volumes of food), we repeated the primary analysis using frequencies alone as the outcome, rather than total amounts consumed. Third, we accounted for possible inattention to the intervention by treating intervention assignment as an instrumental variable for passing the attention check 64] ${ }^{k}$

\subsection{Results}

\subsubsection{Participant characteristics}

We randomized 649 participant: $]^{l}$ at $T_{0}$ (327 in the intervention group and 322 in the control group; Table 1). The sample was roughly balanced on sex, and compared to overall United

\footnotetext{
${ }^{j}$ Other race categories contained few individuals (Table 1 ).

${ }^{k}$ We did not conduct analyses that controlled for passing the attention check, nor did we conduct subset analyses using attentive participants. Both types of analyses are highly problematic; by controlling for a post-intervention variable, they do not estimate any interpretable treatment effect [65]. For example, such analyses do not estimate the treatment effect among attentive participants.

${ }^{l}$ Due to a technical glitch, one participant had a duplicated record in which they had completed the questionnaire twice. We excluded this participant's second record without compromising intention-to-treat principles because the duplicate record did not represent a unique participant.
} 
States national demographics [66], was somewhat younger (median 31 years), more educated (with 58\% having at least graduated from a 4-year college), and considerably more politically liberal (49\% Democrats versus 24\% Republicans). At $T_{1}, 574$ participants completed data collection ( $88 \%$ retention); individual follow-up times between $T_{0}$ and $T_{1}$ had a mean and median of 12 days. Retention was nearly identical for the intervention and control groups ( $88 \%$ and $89 \%$ respectively). A plurality of participants (49\%) indicated that the SARS-CoV-2 pandemic had not changed their ability to choose what they eat.

\subsubsection{Attention check and awareness of study's purpose}

Among intervention-group participants, 95\% correctly indicated that the intervention had discussed farm animal welfare concerns while possibly also indicating other incorrect answers; in contrast, only $14 \%$ of participants in the control group chose this answer. As a more stringent consideration, $76 \%$ of participants in the intervention group chose the single correct answer and no others (i.e., they passed the attention check), compared to $9 \%$ of control participants. Only $3 \%$ of intervention-group participants were potentially aware of the purpose of the study, which was similar to the proportion of participants in the control group who guessed correctly $(1 \%)$.

\subsubsection{Effect of the documentary on outcomes}

Table 2 shows the documentary's estimated effects on all outcomes. The documentary did not reduce total 1-week consumption compared to the control video $(-0.33$ oz/week; $95 \%$ $\mathrm{CI}:[-6.12,5.46] ; p=0.91 ;$ standardized mean difference $[S M D]=-0.01 ; 95 \% \mathrm{CI}:[-0.17$, 0.15]). The documentary also did not meaningfully affect any of the secondary food outcomes or the exploratory attitude outcomes: most standardized mean differences were very close to zero and all were less than 0.20 in magnitude. None of Bonferroni-corrected secondary outcome $p$-values was less than 0.05. As outcome-wide measures of the intervention's effect, the harmonic mean $p$-values were $p=0.66$ for all secondary outcomes considered together, 
$p=0.90$ for the secondary food outcomes, and $p=0.23$ for the exploratory attitude outcomes. Given these null results, we did not pursue cost-effectiveness analyses that we had planned.

\subsubsection{Moderators}

Table 3 shows estimated differences in the documentary's effectiveness for each candidate moderator, along with all main-effect estimates. The results did not support moderation by these variables, at least when considered individually. Demographic characteristics whose estimate direction was consistent with improved intervention effectiveness were, in descending order of estimate magnitude: living in a more politically liberal county (by 10 percentage points), being female, having completed at least 2-year college, being a Democrat (vs. a Republican), being Caucasian, being politically Independent/other, and being $\leq 25$ years old. These findings regarding sex and education [44] and political liberalism [45] are directionally consistent with previous literature.

\subsubsection{Sensitivity analyses}

Conducting complete-case analyses on only the 574 participants who completed data collection at $T_{1}$ yielded similar results to using multiple imputation (Supplementary Table S1). The intervention also did not change participants' reported total frequencies (rather than total weights of foods) of consuming MAP ( -0.34 consumption instances per week; $95 \%$ CI: $[-1.81$, 1.14]; $p=0.65 ; S M D=-0.04 ; 95 \%$ CI: $[-0.19,0.12])$. The instrumental variables analysis suggested that the intervention might have been effective among those participants who passed the attention check $\mathrm{m}^{m}$ (estimated intervention effect of -8.78 oz/week; $95 \%$ CI: [-17.28, $-0.27] ; p=0.043 ; S M D=-0.24 ; 95 \%$ CI: $[-0.48,-0.01])$. Because the primary analyses did not indicate an intervention effect, we did not pursue a planned sensitivity analysis

${ }^{m}$ This analysis makes the excludability assumption [64], namely that if the intervention affected an individual participant's consumption, it did so by first causing the participant to become aware of the documentary's content, as measured by the attention check item. This assumption entails, for example, that the documentary did not have any entirely subconscious means of affecting consumption that in no way required participants to be aware of the documentary's contents. 
283

284

regarding the severity of social desirability bias that would be required to explain away the effect.

\subsection{Discussion}

These null results stand in contrast to those of recent reviews and meta-analyses [10, 16, 17]. The discrepancy could reflect a genuine difference in effectiveness if, for example, the specific documentary we tested was less effective than existing interventions, or because the participants we recruited were less receptive to its effects. Alternatively, the discrepancy could be spurious, reflecting differences in biases affecting our estimates versus those in existing studies. Most existing studies measured outcomes in terms of participants' immediate, reported intentions to reduce consumption, but immediate intentions may not accurately predict actual consumption for at least 2 reasons [17]. First, participants may not follow through on their genuine intentions [67]. Second, when participants are aware of the study's purpose, the intervention might bias some participants' reported intentions (i.e., social desirability bias). We sought to reduce these biases by measuring outcomes after 12 days and using naïve re-recruitment; our analyses suggested that these precautions did successfully blind participants to the study's purpose. To specifically investigate the extent to which these biases might have contributed to the discrepancy in results, we conducted Study 2, which was designed to resemble the majority of existing studies. 


\subsection{Methods}

\subsubsection{Study design, participants, and sample size determination}

We recruited 300 participants on Prolific The recruitment strategy, study design, and sample size determination were identical to the Study 1 except for 2 key differences. First, we collected outcome data immediately after showing participants the documentary or control videos. Second, consistent with the immediate assessment of outcomes, participants reported their intentions using a single item similar to those used in existing studies [26, 68-70]: "How is your consumption of meat and animal products likely to change over the next 7 days?" Participants answered using a 7-point Likert scale ranging from "strongly decrease" to "strongly increase". We reasoned that if the documentary were to remain ineffective when using this outcome measure, then presumably the documentary was genuinely less effective in our Study 1 than similar interventions were in existing studies (e.g., due to characteristics of the intervention itself or differences in the samples studied). On the other hand, if the documentary were to appear more effective when using this outcome measure, this might instead suggest that immediate intentions are not accurate proxies for consumption in the context of intervention studies on reducing MAP consumption.

Following this new primary outcome item, participants answered all items from Study 1's follow-up wave. Critically, the food consumption items still asked participants to report their consumption over the past week, even though Study 2 assessed outcomes immediately after exposure to the assigned video. We reasoned that any apparent intervention effects on these self-reported measures regarding behavior before random assignment and exposure to the documentary or control video would suggest social desirability bias.

\footnotetext{
${ }^{n}$ We chose this sample size a priori to yield $80 \%$ power to detect an effect size of $S M D=0.32$ and $95 \%$ power to detect an effect size of $S M D=0.42$.
} 


\subsubsection{Statistical analyses}

We repeated the analyses of Study 1, omitting sensitivity analyses. We analyzed the new intention outcome on 2 scales: treated as pseudo-continuous $(-3=$ "strongly increase" to 3 = "strongly decrease", with 0 representing no change) and also dichotomized to represent intending to reduce consumption versus intending to increase or not change consumption. The latter binary measure provides an effect size whose scale is directly comparable to the results of a previous meta-analysis [17]. For the continuous intention measure, we calculated a mean difference and estimated inference with a 2-sample Welch's $t$-test; for the binary measure, we calculated a risk ratio and estimated Wald-type inference.

\subsection{Results}

We randomized 300 participants (148 to the documentary and 152 to the control video) and made no exclusions. The participants were demographically very similar to those in Study 1, as expected given the identical recruitment strategies (Supplementary Table S2). Participants in the control group on average reported that they did not intend to change their consumption after watching the control video (0.23 points on the Likert scale from -3 to 3), whereas participants in the intervention group on average reported that they intended to "somewhat decrease" consumption after watching the documentary (0.99 points). Thus, the documentary increased participants' reported intentions to reduce MAP consumption by 0.76 points (95\% CI: [0.49, 1.02]; $p<0.0001 ; S M D=0.65 ; 95 \%$ CI: [0.41, 0.88]) compared to the control video. Similarly, $20 \%$ of participants in the control group reported that they intended to reduce consumption, compared to $68 \%$ in the intervention group. Thus, the documentary increased the percentage of participants intending to reduce consumption by 3.42-fold (95\% CI: [2.49, 4.92]; $p<0.0001)$. As in Study 1, there was little indication of moderation, although Study 2 was not well-powered to detect such effects (Supplementary Table S3). 
Regarding social desirability bias, the documentary did not substantially affect participants' reported total consumption over the week before random assignment $(-4.07$ oz/week; 95\% CI: $[-11.74,3.60] ; p=0.30 ; S M D=-0.12 ; 95 \%$ CI: $[-0.35,0.11])$, though the point estimate was in the direction consistent with social desirability bias. Estimates for individual foods appear in Supplementary Table S4.

\subsection{Discussion}

Whereas Study 1 suggested that the documentary had little, if any, effect on participants' reported consumption at a 12-day follow-up, Study 2 suggested that the documentary substantially increased participants' immediate intentions to reduce consumption. Given the nearly identical sampling frames, a likely explanation is that the documentary did affect participants' stated intentions to reduce consumption, but these intentions had little influence on subsequent self-reported behavior. Alternatively, intentions assessed immediately after the intervention might be particularly susceptible to social desirability bias.

In Study 3 below, we used a design similar to that of Study 1. We enhanced the intervention by adding additional questionnaire items to increase participants' engagement with the documentary content. Additionally, rather than recruiting participants on Prolific, we recruited from a university registry of participants in nutrition research. We speculated that the registry participants might be more receptive to the intervention and attentive than Prolific users. 


\subsection{Methods}

\subsubsection{Study design, participants, and sample size determination}

The study design was similar to that of Study 1, with the following differences. First, we recruited participants from a Stanford University Prevention Research Center registry comprising previous participants of nutrition studies who had either previously participated in nutrition studies or had previously inquired about participating in such research. We invited all 8,346 members of the registry to participate in the $T_{0}$ questionnaire. At $T_{1}$ (14 days after $T_{0}$ ), we invited all participants who had completed $T_{0}$ to complete the $T_{1}$ questionnaire containing the outcome measures. ${ }^{0}$ As in Study 1, we again used naïve re-recruitment (i.e., describing the $T_{1}$ questionnaire as if it were an unrelated study) as a precaution against social desirability bias.

Second, we examined moderation by participant demographics in a confirmatory rather than exploratory manner. In Study 1, we had estimated that 2 demographic characteristics that were directionally consistent with increased intervention effects were having completed at least 2-year college and being a Democrat In Study 2, we hypothesized that the intervention might be more effective for participants with these 2 characteristics (henceforth participants in the "target demographic"). To improve power, we stratified randomization on whether a participant was in the target demographic.

${ }^{\circ}$ We sent the invitation emails at $T_{0}$ in batches over the course of several weeks due to restrictions on the number of emails we could send at once, but we ensured that each participant in the $T_{0}$ questionnaire received the invitation to participate in $T_{1} 14$ days after the date they completed $T_{0}$.

${ }^{p}$ We chose these 2 moderators because, in our initial analyses for Study 1, the moderation estimates were strongest for these 2 variables. After collecting data for all 3 studies, we finalized all the analysis code. During this process, we made modifications to the multiple imputation model to resolve warnings we received from a newly updated version of the imputation software package. (Specifically, we had originally performed imputation on the raw data before creating derived variables such as total consumption, whereas in the final analyses, we calculated the derived variables first before performing imputation.) The resulting, final moderation estimates differed somewhat from those we obtained in the initial analyses. In the final analyses, having completed least 2-year college and being a Democrat were still directionally consistent with increased intervention effects but no longer had the strongest estimates of all moderators we investigated. 


\subsubsection{Intervention}

In this study, we also enhanced the intervention by introducing new questionnaire items (Supplement) designed to increase participant engagement with the documentary content. We presented the documentary as 3 sequential segments corresponding to content about health, the environment, and animal welfare, and presented in the same order as in the original intervention. Each segment was followed by free-response questions about the content presented (e.g., "As discussed in the video, plant-based diets can have several health benefits. Can you name at least 2 of these benefits that you found compelling?").

After the documentary, but not the control video, we asked participants whether they would like to pledge to reduce their consumption, pledge to eliminate their consumption, or to make no pledge regarding their consumption of each of 7 categories of meats and animal products. These pledges conceptually resembled those of a previous study, in which willingness to take certain pledges mediated the intervention effect [71]. To additionally invoke social norms [22, 23, 72], this questionnaire item also stated that "Many of our previous Stanford research participants have pledged to eat and drink less meat and animal products after watching this documentary", a statement that appeared to be true based on pilot studies.

For participants who were assigned to view the documentary and who had chosen to make at least one "reduce" or "eliminate" pledge (henceforth "pledge-making participants"), we then presented several items designed to improve general and specific goal-setting and self-monitoring, which can be effective components of lifestyle behavior-change interventions in general [73]. These items asked pledge-making participants to choose a specific date by which they intended to meet their pledge goals, asked them to select specific strategies they intended to use to fulfill their pledge (e.g., "I will choose a specific day of the week (e.g., Monday) when I will eat only plant-based meals"), and finally suggested concrete ways that participants could track their progress meeting their pledge goals (e.g., smartphone apps). One week after $T_{0}$, we sent a customized email to pledge-making participants. Based on each participant's previous questionnaire responses, this email reminded the participant of the 
414

specific food(s) they had chosen to reduce or eliminate from their diet, of the date on which they intended to meet their pledge goals, and of the strategies they intended to try. These emails were informed by literature on lifestyle behavior-change interventions, which suggests the importance of prompting participants to review their behavioral goals and to monitor their progress toward meeting these goals [73].

\subsubsection{Outcome measures}

The primary and secondary outcome measures were the same as in Study 1. That is, the primary outcome was self-reported MAP consumption, as assessed 14 days after random assignment $\left(T_{1}\right)$ on a food frequency questionnaire.

\subsubsection{Statistical analyses}

The analyses were as in Study 1, with the following changes. In all analyses, we statistically controlled for the variables used to stratify randomization to obtain correct statistical inference (except in models that contained only participants in the target demographic, described below) [74. To do so, we used regression with heteroskedasticity-consistent robust standard errors. These regression models included covariates representing intervention assignment and being in the target demographic.

We also conducted 2 analyses to examine moderation by membership in the target demographic. For the first analysis, we revised the primary analysis model to include an interaction term of membership in the target demographic with intervention assignment. For the second analysis, we estimated the intervention effect within only participants in the target demographic. 


\subsection{Results}

\subsubsection{Participant characteristics}

We randomized 665 participants (333 to the documentary and 332 to the control video) and made no exclusions. ${ }^{q}$ Table 4 shows their demographic characteristics. Compared to the participants of Studies 1 and 2, these participants were more frequently female (73\%), older (median 59 years), more highly educated (with $83 \%$ having at least graduated from a 4-year college), and more politically liberal (60\% Democrats and 4\% Republicans).

At $T_{1}, 217$ participants completed data collection (33\% retention). This was a lower retention rate than was achieved in Study 1A, although retention in this study remained comparable for the intervention and control groups (30\% and 35\% respectively). To explore whether retention was related to participant demographics, we regressed an indicator for retention at $T_{1}$ on all demographic characteristics collected at $T_{0}$ as well as intervention group. This model did not strongly predict retention $(p=0.13)$, suggesting that retention did not differ substantially across participant demographics.

\subsubsection{Attention check and awareness of study's purpose}

At the end of $T_{0}$, almost all participants in the intervention group (98\%) correctly indicated that the intervention had discussed farm animal welfare concerns while possibly also indicating other incorrect answers; no participants in the control group chose this answer. (For comparison, Study 1 found $95 \%$ and 14\%, respectively.) More stringently, $92 \%$ of participants in the intervention group chose the single correct answer and no others (i.e., passed the attention check), compared to $0 \%$ of control participants. (Study 1 found $76 \%$ and 9\%, respectively.) Regarding potential social desirability bias, $13 \%$ of participants in the intervention group were potentially aware of the purpose of the study, versus $5 \%$ of participants in the control group who guessed correctly.

\footnotetext{
${ }^{q}$ Previous research using this university registry has had comparable response rates $(<10 \%)$ to the initial invitation to participate [75].
} 


\subsubsection{Effect of the documentary on outcomes}

The enhanced intervention did not meaningfully reduce total 1-week consumption compared to the control video $(-2.46 \mathrm{oz} /$ week; $95 \% \mathrm{CI}:[-8.78,3.85] ; p=0.43 ; S M D=-0.09 ; 95 \%$ CI: $[-0.32,0.14])$; however, the fairly wide confidence intervals indicated moderate uncertainty. The enhanced intervention also did not appear to affect any of the secondary food outcomes or the exploratory attitude outcomes (Table 5): most standardized mean differences were close to zero and all were less than 0.15. All secondary outcome $p$-values were 1 after Bonferroni correction. The harmonic mean $p$-values were $p=0.95$ for all secondary outcomes considered together, $p=0.86$ for the secondary food outcomes, and $p=0.86$ for the exploratory attitude outcomes.

\subsubsection{Effect of the documentary among participants with target demographics}

The intervention was also not effective among the subset of participants in the target demographic $(-1.72$ oz/week; 95\% CI: [-8.84, 5.41]; $p=0.63 ; S M D=-0.07 ; 95 \% \mathrm{CI}$ : $[-0.34,0.21]$. Corroborating this, the estimated interaction between membership in the target demographic and intervention assignment was small and in the unexpected direction (1.55 oz/week; 95\% CI: $[-7.76,10.85] ; p=0.74 ; S M D=0.06 ; 95 \%$ CI: $[-0.28,0.40])$. However, the confidence intervals for this analysis were quite wide.

\subsubsection{Intervention engagement items}

Of the 332 intervention-group participants, $36 \%$ pledged to eliminate consumption of at least one meat or animal product, $57 \%$ made at least one pledge to reduce consumption, and $60 \%$ made at least one pledge of either type. Table 6 disaggregates percentages by pledge type and food type. 


\subsubsection{Sensitivity analyses}

Complete-case analyses $(n=217)$ of participants who completed data collection at $T_{1}$ yielded a somewhat stronger intervention effect than primary analyses (Supplementary Table S5). An instrumental variables analysis suggested that the intervention effect among attentive participants was approximately twice as strong as that seen in the primary analysis $(-5.33$ oz/week; 95\% CI: $[-14.44,3.78] ; p=0.24 ; S M D=-0.20 ; 95 \%$ CI: $[-0.53,0.14])$, but the confidence interval was very wide.

\subsection{Discussion}

This study corroborated the results of Study 1, this time with an enhanced intervention and in a new population of participants, who were more attentive and likely more strongly motivated by health considerations $I^{T}$ Based on our moderator analysis from Study 1, these participants' demographics also suggested that they might be more receptive to the intervention. However, even in these favorable conditions, we did not observe an improvement in the enhanced intervention's effectiveness. Additionally, there was little evidence that the intervention was effective even among participants in the target demographic. The modest retention rate in this study tempers conclusions, although it is somewhat reassuring that intervention assignment and demographics did not strongly predict retention. Additionally, participants at $T_{1}$ were still largely unaware of the purpose of the study. We used statistical methods that can reduce some, but not all, forms of bias due to missing data; nevertheless, missing data still reduces statistical power.

\section{General Discussion}

In a series of 3 randomized, controlled experiments, we investigated the effectiveness of a documentary that was professionally designed to reduce MAP consumption using many

${ }^{r}$ Among only control-group participants, average ratings for the importance of health (which was one of the exploratory attitude outcomes) were 4.23 in Study 3 versus 3.88 in Study 1. 
504

of the best practices in behavior-change intervention design. The documentary's content was designed to leverage general components of effective behavioral interventions (e.g., by providing educational information, invoking social norms, and providing implementation suggestions) and of the specific psychology governing the MAP consumption (e.g., by invoking physical and moral disgust and using meat-animal reminders).

In a study designed to minimize social desirability bias (Study 1), we found that the documentary did not affect participants' reported consumption 12 days after random assignment (-0.33 oz/week; 95\% CI: [-6.12, 5.46]; Study 1). However, in a second study with less protection against social desirability bias (Study 2), the documentary did substantially increase the percentage of participants who immediately intended to reduce consumption from $20 \%$ to $68 \%$, a 3.42-fold change (95\% CI: [2.49, 4.92]; Study 2), consistent with the consensus of existing studies and meta-analyses. Finally, we changed the study sample from members of a commercial study recruitment platform to participants who had previously volunteered to be contacted about nutrition studies and enhanced the intervention with evidence-based engagement items and opportunities to form concrete goals (Study 3). These changes did not markedly improve the intervention's effects on subsequent reported MAP consumption $(-2.46$ oz/week; 95\% CI: [-8.78, 3.85]), even though a majority of participants who viewed the documentary $(60 \%)$ had pledged to reduce or eliminate their consumption of at least one meat or animal product. Additionally, the documentary was not substantially more effective among participants who were expected to be more receptive to its content: among participants who had expressed interest in participating in nutrition studies (all participants in Study 3) or among the demographic subset of these participants who were Democrats and had at least graduated 2-year college. The documentary also did not meaningfully affect participants' responses to secondary consumption outcomes or the exploratory attitude outcomes. 


\subsection{Strengths and limitations}

This research has a number of methodological and conceptual strengths. To help mitigate widespread limitations of previous research regarding the potential for social desirability bias [17], we introduced methodological innovations to help prevent and benchmark social desirability bias in studies of reported food outcomes. First, in the 2 longitudinal studies (Studies 1 and 3), we used naïve re-recruitment to conceal the purpose of the study. We also included decoy foods (e.g., sweetened beverages) in the outcome measurements. Awareness probes in these studies indicated that, with these methods, participants indeed remained largely unaware of the purpose of the experiment during follow-up data collection. In Study 2 , as a benchmark of the severity of social desirability bias, we investigated "retrospective causation" by asking participants to report on their past-week consumption, mere minutes after they had viewed either the documentary or the control video. Any apparent intervention effects on reported consumption over the week prior to random assignment would appear to reflect social desirability bias. We estimated that the documentary affected these retrospective reports of total consumption by $-4.07 \mathrm{oz} /$ week $(95 \% \mathrm{CI}$ : $[-11.74,3.60])$. Although the confidence interval is wide, this point estimate is in the vicinity of typical intervention effects seen in studies of interventions to reduce meat consumption, most of which had similar designs that are susceptible to social desirability bias [17].

As an additional methodological strength, in Studies 1 and 3, we assessed reported consumption rather than only intentions, and we assessed consumption in terms of the numerical weight of food that participants reported consuming of specific categories of meats and animal products (e.g., chicken, beef, etc.). Such measures would allow intervention effects to be translated into direct measures of cost-effectiveness and societal impact, such as the estimated reduction in human all-cause mortality events, in the number of animals raised for consumption, and in greenhouse gas emissions [17]. Last, in Study 3, we enhanced the intervention with engagement items that were based on strong evidence from the broader 
literature on lifestyle behavior-change interventions, including tailored content via the interim emails reminding participants of their stated pledges [73]. These enhancements have rarely been studied in the context of reducing MAP consumption [17].

This research also has limitations. Although our findings seem to rule out the possibility of large intervention effects, smaller intervention effects may nevertheless be consequential if a large number of individuals were exposed to the intervention, or if effects accumulated over repeated exposures [76]. Indeed, we intentionally selected an intervention that has already been widely disseminated at relatively low cost. However, it is difficult to precisely establish small intervention effects without very large sample sizes and high retention. Although Study 1 achieved high retention, Study 3 did not, which compromises statistical precision and increases susceptibility to missing data bias. Additionally, reported dietary data can be subject to substantial measurement error even when collected via measures such as 24-hour recalls or food frequency questionnaires [77, 78]; such measurement error can further obscure small intervention effects. Although Studies 1 and 2 used somewhat longer durations of follow-up (approximately 2 weeks) than most existing studies [17], even longer durations would help identify potential delayed adoption and would characterize how well effects are sustained over time. The documentary we studied was previously disseminated by a nonprofit, so it is possible that some individuals may already have viewed it prior to participating in our studies, although no participants mentioned having seen the documentary when they provided free-text feedback on the study. Finally, it is possible that the control video could have elicited effects of its own, although this seems unlikely given its irrelevant content.

\subsection{Future directions}

The majority of previous studies of similar interventions have assessed outcomes in terms of participants' reported intentions to change their consumption, using designs similar to our Study 2 [17]. Indeed, in this context, the documentary we studied appeared to be remarkably effective, with an effect size stronger than those of all 100 studies included in our recent 
580

meta-analysis [17] (i.e., in Study 2, we estimated a 3.42-fold reduction in the percentage of participants who intended to reduce consumption). Critically, though, the documentary had little effect on reported consumption after approximately 2 weeks. This discrepancy between intentions and reported consumption reiterates that reported intentions may be a poor proxy for reported actual consumption, and further underscores the importance of designing studies that minimize social desirability bias. Future longitudinal studies could consider adopting the same methodological innovations, detailed above, that we used to successfully reduce participant awareness.

As noted above, precisely estimating potentially small, but consequential, intervention effects requires large sample sizes as well as stringent precautions against biases and sources of random measurement error. Future studies of similar interventions might consider using designs other than traditional individual randomization. For example, interventions could be deployed at locations of food purchases (e.g., educational flyers posted in a grocery store), which could potentially take advantage of take advantage of social norms generated within groups and also facilitate measuring outcomes in terms of actual food purchases at that location. Such designs can enable large sample sizes while reducing the potential for social desirability bias and measurement error [79].

In conclusion, these findings suggest that some past studies of similar interventions may have overestimated effects due to methodological biases. The methodological innovations we introduced could help future studies mitigate these biases. Novel intervention strategies may be needed to effectively shift dietary consumption away from MAP. 


\section{ACKNOWLEDGMENTS}

We thank Jennifer Robinson, Antonella Dewell, and Vincent Busque for assisting with data collection and Adam Peditto for directing the Good For Us documentary.

\section{AVAilability OF DATA AND MATERIALS}

The datasets supporting the conclusions of this article are available in the Open Science Framework repository (DOI 10.17605/osf.io/xrckh; https://osf.io/8dzng/) as commaseparated value (CSV) files. Participants' counties of residence are omitted from the datasets per our IRB approval. The documentary and control video, questionnaire materials, and analysis code are also publicly available and documented (https://osf.io/8dzng/). The documentary intervention is freely available under copyright by The Humane League. Others wishing to reuse the intervention for research or educational purposes are encouraged to secure permission from The Humane League, though such uses are typically covered under fair use in the United States.

\section{ETHICS APPROVAL AND CONSENT TO PARTICIPATE}

All studies were approved by the Stanford University IRB (protocol \#57476).

\section{COnSEnt FOR PUBlicAtion}

Not applicable.

Maya B. Mathur is a member of the Research Advisory Board of the Better Food Foundation. Christopher D. Gardner received gift funding from Beyond Meat to create a Plant-Based 
621

Diet Initiative at Stanford University. Jacob R. Peacock is affiliated with The Humane League Labs (THLL). THLL performs scientific research to inform animal advocacy strategy. THLL is a program of The Humane League (THL), a 501(c)(3) nonprofit organization that "exists to end the abuse of animals raised for food." THLL is editorially independent from THL, and any other funders, in reporting research results. The design, execution, analysis, interpretation, and reporting of THLL research is performed entirely by THLL staff, without oversight by other THL staff or leadership. Thomas N. Robinson is a member of Scientific Advisory Boards for WW International, Inc.

\footnotetext{
This research was supported by the Food Systems Research Fund (award 2-019). The funders played no role in the interpretation of these results or the decision to publish.
}

\section{FUNDING}

\section{Authors' CONTRIBUtions}

All authors conceived and designed the study. MBM collected data, conducted statistical analyses, and led writing of the manuscript. All authors contributed critical input to writing and interpretation, and have approved of the final draft.

\section{LIST OF ABBREVIATIONS}

- $T_{0}$ : The baseline wave of data collection

- $T_{1}$ : The follow-up wave of data collection

- CI: Confidence interval

- SMD: Standardized mean difference (Hedges' g) 
[1] Walter Willett, Johan Rockström, Brent Loken, Marco Springmann, Tim Lang, Sonja Vermeulen, Tara Garnett, David Tilman, Fabrice DeClerck, Amanda Wood, et al. Food in the Anthropocene: the EAT-Lancet Commission on healthy diets from sustainable food systems. The Lancet, 393(10170):447-492, 2019.

[2] Boyd A Swinburn, Vivica I Kraak, Steven Allender, Vincent J Atkins, Phillip I Baker, Jessica R Bogard, Hannah Brinsden, Alejandro Calvillo, Olivier De Schutter, Raji Devarajan, et al. The global syndemic of obesity, undernutrition, and climate change: the Lancet Commission report. The Lancet, 393(10173):791-846, 2019.

[3] Christopher D Gardner, Jennifer C Hartle, Rachael D Garrett, Lisa C Offringa, and Arlin S Wasserman. Maximizing the intersection of human health and the health of the environment with regard to the amount and type of protein produced and consumed in the united states. Nutrition Reviews, 77(4):197-215, 2019.

[4] H Charles J Godfray, Paul Aveyard, Tara Garnett, Jim W Hall, Timothy J Key, Jamie Lorimer, Ray T Pierrehumbert, Peter Scarborough, Marco Springmann, and Susan A Jebb. Meat consumption, health, and the environment. Science, 361(6399):eaam5324, 2018.

[5] David Tilman and Michael Clark. Global diets link environmental sustainability and human health. Nature, 515(7528):518-522, 2014.

[6] Laura Scherer, Paul Behrens, and Arnold Tukker. Opportunity for a dietary win-win-win in nutrition, environment, and animal welfare. One Earth, 1(3):349-360, 2019.

[7] Moreno Di Marco, Michelle L Baker, Peter Daszak, Paul De Barro, Evan A Eskew, Cecile M Godde, Tom D Harwood, Mario Herrero, Andrew J Hoskins, Erica Johnson, 
et al. Opinion: Sustainable development must account for pandemic risk. Proceedings of the National Academy of Sciences, 117(8):3888-3892, 2020.

[8] Intergovernmental Panel on Climate Change. Climate change 2021: The physical science basis. Contribution of Working Group I to the Sixth Assessment Report of the Intergovernmental Panel on Climate Change. Technical report, 2021. Retrieved from $<$ https://www ipcc.ch/report/ar6/wg1/downloads/report/IPCC_AR6_WGI_Full_Report.pdf $>$ on 2021-9-6.

[9] Céline Bonnet, Zohra Bouamra-Mechemache, Vincent Réquillart, and Nicolas Treich. Regulating meat consumption to improve health, the environment and animal welfare. Food Policy, page 101847, 2020.

[10] Filippo Bianchi, Claudia Dorsel, Emma Garnett, Paul Aveyard, and Susan A Jebb. Interventions targeting conscious determinants of human behaviour to reduce the demand for meat: A systematic review with qualitative comparative analysis. International Journal of Behavioral Nutrition and Physical Activity, 15(1):102, 2018.

[11] Stephen S Lim, Theo Vos, Abraham D Flaxman, Goodarz Danaei, Kenji Shibuya, Heather Adair-Rohani, Mohammad A AlMazroa, Markus Amann, H Ross Anderson, Kathryn G Andrews, et al. A comparative risk assessment of burden of disease and injury attributable to 67 risk factors and risk factor clusters in 21 regions, 1990-2010: a systematic analysis for the global burden of disease study 2010. The lancet, 380(9859):2224-2260, 2012.

[12] Pramil N Singh, Joan Sabaté, and Gary E Fraser. Does low meat consumption increase life expectancy in humans? The American Journal of Clinical Nutrition, 78(3):526S-532S, 2003.

[13] P Gerber, C Opio, and H Steinfeld. Poultry production and the environment-a review. Technical report, Animal Production and Health Division, Food and Agriculture 
Organization of the United Nations, 2007. http://www.fao.org/ag/againfo/home/ events/bangkok2007/docs/part2/2_2.pdf

[14] United Nations General Assembly. The impacts of fishing on vulnerable marine ecosystems: Actions taken by states and regional fisheries management organizations and arrangements to give effect to paragraphs 66 to 69 of general assembly resolution $59 / 25$ on sustainable fisheries, regarding the impacts of fishing on vulnerable marine ecosystems. Technical report, United Nations, November 2016. https: //www.un.org/Depts/los/general_assembly/documents/impact_of_fishing.pdf.

[15] Marten Scheffer, Steve Carpenter, and Brad de Young. Cascading effects of overfishing marine systems. Trends in Ecology \& Evolution, 20(11):579-581, 2005.

[16] Emily Grundy, Peter Slattery, Alexander K Saeri, Kieren Watkins, Thomas Houlden, Neil Farr, Henry Askin, Joannie Lee, Alexandria Mintoft-Jones, Sophia Cyna, et al. Interventions that influence animal-product consumption: A meta-review. Future Foods, 2021. Preprint retrieved from https://osf.io/mcdsq/

[17] Maya B Mathur, Jacob Peacock, David B Reichling, Janice Nadler, Paul A Bain, Christopher D Gardner, and Thomas N Robinson. Interventions to reduce meat consumption by appealing to animal welfare: Meta-analysis and evidence-based recommendations. Appetite, 164:105277, 2021.

[18] Filippo Bianchi, Emma Garnett, Claudia Dorsel, Paul Aveyard, and Susan A Jebb. Restructuring physical micro-environments to reduce the demand for meat: A systematic review and qualitative comparative analysis. The Lancet Planetary Health, 2(9):e384e397, 2018.

[19] James R Hebert, Lynn Clemow, Lori Pbert, Ira S Ockene, and Judith K Ockene. Social desirability bias in dietary self-report may compromise the validity of dietary intake measures. International Journal of Epidemiology, 24(2):389-398, 1995. 
[20] Icek Ajzen et al. The theory of planned behavior. Organizational Behavior and Human Decision Processes, 50(2):179-211, 1991.

[21] Marleen C Onwezen and Cor N van der Weele. When indifference is ambivalence: Strategic ignorance about meat consumption. Food Quality and Preference, 52:96-105, 2016. https://doi.org/10.1016/j.foodqual.2016.04.001.

[22] P Wesley Schultz, Jessica M Nolan, Robert B Cialdini, Noah J Goldstein, and Vladas Griskevicius. The constructive, destructive, and reconstructive power of social norms. Psychological Science, 18(5):429-434, 2007. https://doi.org/10.1111/j.14679280.2007.01917.x.

[23] Suzanne Higgs. Social norms and their influence on eating behaviours. Appetite, 86:38-44, 2015. https://doi.org/10.1016/j.appet.2014.10.021.

[24] Marieke A Adriaanse, Charlotte DW Vinkers, Denise TD De Ridder, Joop J Hox, and John BF De Wit. Do implementation intentions help to eat a healthy diet? A systematic review and meta-analysis of the empirical evidence. Appetite, 56(1):183-193, 2011.

[25] Catherine E Amiot, Guy El Hajj Boutros, Ksenia Sukhanova, and Antony D Karelis. Testing a novel multicomponent intervention to reduce meat consumption in young men. PloS One, 13(10):e0204590, 2018.

[26] BNJ Macdonald, KD Caldwell, and GD Boese. The effects of 'reduce' and 'eliminate' appeals on individual meat consumption. Technical report, Reducetarian Foundation, 2016. https://osf.io/nxrx3/.

[27] Adéla Novatná. The Influence of movie on behavioral change in individual meat and dairy products consumption. Unpublished bachelor thesis, Masaryk University, Brno, Czech Republic, April 2019. https://is.muni.cz/th/cfyr2/Novotna_bakalarska_prace.pdf. 
[28] Steve Loughnan, Brock Bastian, and Nick Haslam. The psychology of eating animals. Current Directions in Psychological Science, 23(2):104-108, 2014.

[29] Paul Rozin. Towards a psychology of food and eating: From motivation to module to model to marker, morality, meaning, and metaphor. Current Directions in Psychological Science, 5(1):18-24, 1996.

[30] Amelia Cornish, David Raubenheimer, and Paul McGreevy. What we know about the public's level of concern for farm animal welfare in food production in developed countries. Animals, 6(11):74, 2016.

[31] Brock Bastian and Steve Loughnan. Resolving the meat-paradox: A motivational account of morally troublesome behavior and its maintenance. Personality and Social Psychology Review, 21(3):278-299, 2017.

[32] Jonas R Kunst and Sigrid M Hohle. Meat eaters by dissociation: How we present, prepare and talk about meat increases willingness to eat meat by reducing empathy and disgust. Appetite, 105:758-774, 2016.

[33] Jonas R Kunst and Christian Andrés Palacios Haugestad. The effects of dissociation on willingness to eat meat are moderated by exposure to unprocessed meat: A cross-cultural demonstration. Appetite, 120:356-366, 2018.

[34] Megan Earle, Gordon Hodson, Kristof Dhont, and Cara MacInnis. Eating with our eyes (closed): Effects of visually associating animals with meat on antivegan/vegetarian attitudes and meat consumption willingness. Group Processes and Intergroup Relations, $22(6): 1-18,2019$.

[35] Qirui Tian, Denis Hilton, and Maja Becker. Confronting the meat paradox in different cultural contexts: Reactions among Chinese and French participants. Appetite, 96:187$194,2016$. 
[36] Catarina RP da Silva. Am I too cute to eat? The effect of cuteness appeal towards the promotion of a more plant-based diet. Master's thesis, University Institute of Lisbon, 2016. http://hdl.handle.net/10071/12583

[37] Zoé Lackner. The effect of dissociation between meat and animal on the experience of ambivalence towards meat. Université de Grenoble Alpes, 2019.

[38] Paul Rozin and April Fallon. The psychological categorization of foods and non-foods: A preliminary taxonomy of food rejections. Appetite, 1(3):193-201, 1980.

[39] Matthew Feinberg, Chloe Kovacheff, Rimma Teper, and Yoel Inbar. Understanding the process of moralization: How eating meat becomes a moral issue. Journal of Personality and Social Psychology, 117:50-72, 2019.

[40] Hanah A Chapman and Adam K Anderson. Things rank and gross in nature: A review and synthesis of moral disgust. Psychological Bulletin, 139(2):300, 2013.

[41] Anette Cordts, Sina Nitzko, and Achim Spiller. Consumer response to negative information on meat consumption in Germany. International Food and Agribusiness Management Review, 17(Special Issue A):83-106, 2014.

[42] Matthew B Ruby. Vegetarianism. A blossoming field of study. Appetite, 58(1):141-150, 2012.

[43] Hank Rothgerber. Meat-related cognitive dissonance: A conceptual framework for understanding how meat eaters reduce negative arousal from eating animals. Appetite, 146:104511, 2020. https://doi.org/10.1016/j.appet.2019.104511.

[44] João Graça, Cristina A Godinho, and Monica Truninger. Reducing meat consumption and following plant-based diets: Current evidence and future directions to inform integrated transitions. Trends in Food Science Eamp; Technology, 91:380-390, 2019. 
[45] Gregg Sparkman, Bobbie NJ Macdonald, Krystal D Caldwell, Brian Kateman, and Gregory D Boese. Cut back or give it up? the effectiveness of reduce and eliminate appeals and dynamic norm messaging to curb meat consumption. Journal of Environmental Psychology, 75:101592, 2021.

[46] R Core Team. R: A Language and Environment for Statistical Computing. R Foundation for Statistical Computing, Vienna, Austria, 2019.

[47] Qualtrics, Provo, UT. Qualtrics XM Platform, 2020.

[48] Prolific, London, UK. Prolific Academic, 2020.

[49] Eyal Peer, Laura Brandimarte, Sonam Samat, and Alessandro Acquisti. Beyond the Turk: Alternative platforms for crowdsourcing behavioral research. Journal of Experimental Social Psychology, 70:153-163, 2017.

[50] Jesse Chandler and Danielle Shapiro. Conducting clinical research using crowdsourced convenience samples. Annual Review of Clinical Psychology, 12, 2016.

[51] The Humane League. Good for us, July 2020.

[52] Diet History Questionnaire III (DHQ III). Technical report, National Cancer Institute, March 2018. https://epi.grants.cancer.gov/dhq3/.

[53] Frances E Thompson, Amy F Subar, Charles C Brown, Albert F Smith, Carolyn O Sharbaugh, Jared B Jobe, Beth Mittl, James T Gibson, and Regina G Ziegler. Cognitive research enhances accuracy of food frequency questionnaire reports: results of an experimental validation study. Journal of the American Dietetic Association, 102(2):212$225,2002$.

[54] Amy E Millen, Douglas Midthune, Frances E Thompson, Victor Kipnis, and Amy F Subar. The National Cancer Institute diet history questionnaire: validation of pyramid food servings. American Journal of Epidemiology, 163(3):279-288, 2006. 
[55] Eric B Hekler, Christopher D Gardner, and Thomas N Robinson. Effects of a college course about food and society on students' eating behaviors. American Journal of Preventive Medicine, 38(5):543-547, 2010.

[56] James H Stapleton. Linear statistical models, volume 719. John Wiley \& Sons, 2009.

[57] Morten W Fagerland. t-tests, non-parametric tests, and large studies - a paradox of statistical practice? BMC Medical Research Methodology, 12(1):78, 2012.

[58] Charles W Dunnett. A multiple comparison procedure for comparing several treatments with a control. Journal of the American Statistical Association, 50(272):1096-1121, 1955.

[59] Daniel J Wilson. The harmonic mean p-value for combining dependent tests. Proceedings of the National Academy of Sciences, 116(4):1195-1200, 2019.

[60] Tyler J VanderWeele, Maya B Mathur, Ying Chen, et al. Outcome-wide longitudinal designs for causal inference: a new template for empirical studies. Statistical Science, 35(3):437-466, 2020.

[61] Tyler J VanderWeele and Maya B Mathur. Some desirable properties of the Bonferroni correction: is the Bonferroni correction really so bad? American Journal of Epidemiology, 188(3):617-618, 2019.

[62] S van Buuren and Karin Groothuis-Oudshoorn. mice: Multivariate imputation by chained equations in R. Journal of Statistical Software, pages 1-68, 2010.

[63] Stef Van Buuren. Flexible imputation of missing data. CRC press, 2018.

[64] Joshua D Angrist, Guido W Imbens, and Donald B Rubin. Identification of causal effects using instrumental variables. Journal of the American Statistical Association, 91(434):444-455, 1996.

[65] Peter M Aronow, Jonathon Baron, and Lauren Pinson. A note on dropping experimental subjects who fail a manipulation check. Political Analysis, 27(4):572-589, 2019. 
[66] U.S. Census Bureau. American Community Survey 1-year estimates, 2019. Retrieved from Census Reporter Profile page for United States $<$ http://censusreporter.org/ profiles/01000US-united-states $>$ on 2021-9-6.

[67] Jonathan van’t Riet, Siet J Sijtsema, Hans Dagevos, and Gert-Jan De Bruijn. The importance of habits in eating behaviour. an overview and recommendations for future research. Appetite, 57(3):585-596, 2011.

[68] Jacy Reese. Testing the effectiveness of animal advocacy messages with Amazon Mechanical Turk. Technical report, The Sentience Institute, March 2015. https://jacyreese.com/assets/downloads/Testing_the_Effectiveness.pdf.

[69] Krystal Caldwell. Welfare reforms and meat consumption. Technical report, Mercy for Animals, November 2016. https://mercyforanimals.org/welfare-reforms-survey.

[70] Gonzalo Palomo-Vélez, Joshua M Tybur, and Mark van Vugt. Unsustainable, unhealthy, or disgusting? Comparing different persuasive messages against meat consumption. Journal of Environmental Psychology, 58:63-71, 2018.

[71] Jo Anderson. "Reduce" or "Go Veg?": Effects on meal choice. Technical report, Faunalytics, 2020. https://faunalytics.org/reduce-or-go-veg/.

[72] Gregg Sparkman, Elizabeth Weitz, Thomas N Robinson, Neil Malhotra, and Gregory M Walton. Developing a scalable dynamic norm menu-based intervention to reduce meat consumption. Sustainability, 12(6):2453, 2020.

[73] Susan Michie, Charles Abraham, Craig Whittington, John McAteer, and Sunjai Gupta. Effective techniques in healthy eating and physical activity interventions: a metaregression. Health Psychology, 28(6):690, 2009.

[74] Brennan C Kahan and Tim P Morris. Improper analysis of trials randomised using stratified blocks or minimisation. Statistics in Medicine, 31(4):328-340, 2012. 
[75] Walter Sobba, Matthew J Landry, Kristen M Cunanen, Alessandra Marcone, and Christopher D Gardner. Changes in ultra-processed food consumption and lifestyle behaviors following COVID-19 shelter-in-place: A retrospective survey. 2021. Under review.

[76] David C Funder and Daniel J Ozer. Evaluating effect size in psychological research: Sense and nonsense. Advances in Methods and Practices in Psychological Science, 2(2):156-168, 2019.

[77] Jacob Peacock. Measuring change in diet for animal advocacy. Technical report, The Humane League Labs, 2018. "https://thehumaneleague.org/article/ E009R01-measuring-diet-animal-advocacy".

[78] Laurence S Freedman, John M Commins, James E Moler, Lenore Arab, David J Baer, Victor Kipnis, Douglas Midthune, Alanna J Moshfegh, Marian L Neuhouser, Ross L Prentice, et al. Pooled results from 5 validation studies of dietary self-report instruments using recovery biomarkers for energy and protein intake. American Journal of Epidemiology, 180(2):172-188, 2014.

[79] Carmen Piernas, Brian Cook, Richard Stevens, Cristina Stewart, Jennifer Hollowell, Peter Scarborough, and Susan A Jebb. Estimating the effect of moving meat-free products to the meat aisle on sales of meat and meat-free products: A non-randomised controlled intervention study in a large UK supermarket chain. PLoS Medicine, 18(7):e1003715, 2021. 
Sex

Male

$164(50 \%)$

$178(55 \%)$

Female

$158(48 \%)$

$140(43 \%)$

Other

$5(2 \%)$

$4(1 \%)$

Age (years)

$30(24,41)$

$32(23,41)$

\section{Education}

Did not graduate high school

Graduated high school

Graduated 2-year college

$0(0 \%)$

$102(31 \%)$

$36(11 \%)$

$116(35 \%)$

$73(22 \%)$

Completed post-graduate degree

\section{Political party}

Democrat

Republican

Independent

Other/I don't know

County liberalism

Race

Caucasian

Black/African American

Hispanic

East Asian

Southeast Asian

South Asian

Native American

Middle Eastern

Pacific Islander
$242(74 \%)$

$32(10 \%)$

$26(8 \%)$

$24(7 \%)$

$9(3 \%)$

$12(4 \%)$

$8(2 \%)$

$2(1 \%)$

$3(1 \%)$
$2(1 \%)$

$103(32 \%)$

$28(9 \%)$

$119(37 \%)$

$70(22 \%)$

$171(53 \%)$

$76(24 \%)$

$61(19 \%)$

$14(4 \%)$

$0.55(0.43,0.70)$

$229(71 \%)$

$25(8 \%)$

$30(9 \%)$

$30(9 \%)$

$13(4 \%)$

$11(3 \%)$

$9(3 \%)$

$6(2 \%)$

$3(1 \%)$

Table 1. For Study 1, demographic characteristics of the 649 participants at baseline. Continuous variables are reported as medians with 25th and 75th percentiles. Binary variables are reported as counts and percentages. Subjects could indicate multiple races. "County liberalism": in the subject's county, the proportion of votes from the 2000-2016 United States presidential elections that went to the Democratic candidate. 


\begin{tabular}{|c|c|c|c|c|}
\hline \multicolumn{5}{|l|}{ Primary outcome } \\
\hline Total meat and animal products & $-0.33[-6.12,5.46]$ & $-0.01[-0.17,0.15]$ & 0.91 & \\
\hline \multicolumn{5}{|l|}{ Secondary food outcomes } \\
\hline Meat & $-1.14[-5.25,2.97]$ & $-0.04[-0.2,0.11]$ & 0.59 & 1 \\
\hline Non-meat animal products & $0.82[-2.43,4.07]$ & $0.04[-0.13,0.21]$ & 0.62 & 1 \\
\hline Turkey & $-0.5[-1.42,0.41]$ & $-0.09[-0.26,0.08]$ & 0.28 & 1 \\
\hline Fish & $0.00[-1.03,1.04]$ & $0.00[-0.16,0.16]$ & 1 & 1 \\
\hline Pork & $-0.1[-0.98,0.78]$ & $-0.02[-0.18,0.14]$ & 0.82 & 1 \\
\hline Beef & $-0.39[-1.62,0.84]$ & $-0.05[-0.21,0.11]$ & 0.53 & 1 \\
\hline Other meat & $-0.16[-0.95,0.64]$ & $-0.03[-0.19,0.13]$ & 0.7 & 1 \\
\hline Dairy & $1.09[-1.72,3.9]$ & $0.07[-0.11,0.24]$ & 0.45 & 1 \\
\hline Importance of health & $0.10[-0.10,0.30]$ & $0.08[-0.08,0.24]$ & 0.34 & 1 \\
\hline Importance of environment & $0.06[-0.16,0.29]$ & $0.05[-0.12,0.21]$ & 0.57 & 1 \\
\hline Importance of animal welfare & $0.18[-0.04,0.39]$ & $0.13[-0.03,0.29]$ & 0.12 & 1 \\
\hline Interest in activism & & $0.17[0.01,0.33]$ & 0.04 & 0.64 \\
\hline Speciesism & & $-0.08[-0.24,0.09]$ & 0.36 & 1 \\
\hline Social dominance orientation & & $-0.03[-0.2,0.13]$ & 0.68 & 1 \\
\hline
\end{tabular}

Table 2. In Study 1, estimated intervention effects for the primary outcome, secondary food outcomes, and exploratory attitude outcomes. Raw mean differences represent ounces consumed over the past week for the primary outcome and secondary food outcomes; they represent units on a 7-point Likert scale for the perceived importance items; and they are omitted for the three composite scales, which were already standardized. Brackets are 95\% confidence intervals without correcting for multiple testing. 


\begin{tabular}{|c|c|c|c|c|}
\hline Coefficient & Raw mean difference & Standardized mean difference & p-value & Bonferroni p-value \\
\hline \multicolumn{5}{|l|}{ Main effects } \\
\hline Intercept & $51.2[28.99,73.41]$ & $1.42[0.8,2.04]$ & $<0.0001$ & \\
\hline Intervention (vs. control) & $16.96[-12.2,46.13]$ & $0.47[-0.34,1.28]$ & 0.25 & \\
\hline Female & $-9[-19.08,1.08]$ & $-0.25[-0.53,0.03]$ & 0.08 & \\
\hline Age $\leq 25$ years & $1.71[-7.13,10.55]$ & $0.05[-0.2,0.29]$ & 0.7 & \\
\hline At least 2 -year college & $2.66[-5.97,11.28]$ & $0.07[-0.17,0.31]$ & 0.54 & \\
\hline Caucasian & $5.66[-2.67,13.98]$ & $0.16[-0.07,0.39]$ & 0.18 & \\
\hline Democrat (vs. Independent/other) & $-0.21[-14.53,14.1]$ & $-0.01[-0.4,0.39]$ & 0.98 & \\
\hline Republication (vs. Independent/other) & $-3.63[-18.24,10.98]$ & $-0.1[-0.51,0.3]$ & 0.63 & \\
\hline County liberalism & $0.9[-2.83,4.62]$ & $0.02[-0.08,0.13]$ & 0.64 & \\
\hline \multicolumn{5}{|l|}{ Moderation of intervention effect } \\
\hline Female & $-2.3[-15.21,10.61]$ & $-0.06[-0.42,0.29]$ & 0.73 & 1 \\
\hline Age $\leq 25$ years & $-0.37[-12.97,12.22]$ & $-0.01[-0.36,0.34]$ & 0.95 & 1 \\
\hline At least 2 -year college & $-2.22[-14.44,9.99]$ & $-0.06[-0.4,0.28]$ & 0.72 & 1 \\
\hline Caucasian & $-0.71[-12.43,11.01]$ & $-0.02[-0.34,0.31]$ & 0.9 & 1 \\
\hline Independent/other (vs. Republican) & $-0.58[-19.07,17.9]$ & $-0.02[-0.53,0.5]$ & 0.95 & 1 \\
\hline Democrat (vs. Republican) & $-0.75[-19.04,17.55]$ & $-0.02[-0.53,0.49]$ & 0.94 & 1 \\
\hline County liberalism & $-2.34[-6.71,2.03]$ & $-0.07[-0.19,0.06]$ & 0.29 & 1 \\
\hline
\end{tabular}

Table 3. For Study 1, estimated moderation by baseline demographic variables of the intervention's effect on the primary outcome (total meat and animal product consumption). Raw mean differences represent ounces consumed over the past week. Main effects represent differences in average consumption by the demographic variables. Effect modification estimates represent differences in intervention effectiveness for each demographic variable, with negative values representing greater effectiveness (i.e., greater reductions in consumption). Brackets are 95\% confidence intervals without multiplicity corrections. "Politically neutral": Independent or "Other/I don't know". "County liberalism" represents a 10-percentage point higher proportion of votes cast for Democratic presidential candidates in the participant's county. 
Sex

Male

$82(25 \%)$

$98(30 \%)$

Female

$251(75 \%)$

$234(70 \%)$

Other

$0(0 \%)$

$0(0 \%)$

Age (years)

$60(48,67)$

$58(49,67)$

\section{Education}

Did not graduate high school

Graduated high school

Graduated 2-year college

$2(1 \%)$

$28(8 \%)$

$32(10 \%)$

$146(44 \%)$

$125(38 \%)$

Completed post-graduate degree

\section{Political party}

Democrat

Republican

Independent

Other/I don't know

\section{County liberalism}

Race

Caucasian

Black/African American

Hispanic

East Asian

Southeast Asian

South Asian

Native American

Middle Eastern

Pacific Islander
$209(63 \%)$

$15(5 \%)$

$61(18 \%)$

$48(14 \%)$

$0.70(0.70,0.74)$

$258(77 \%)$

$6(2 \%)$

$26(8 \%)$

$23(7 \%)$

$18(5 \%)$

$12(4 \%)$

$3(1 \%)$

$2(1 \%)$

$5(2 \%)$
$2(1 \%)$

$24(7 \%)$

$26(8 \%)$

$127(38 \%)$

$153(46 \%)$

$192(58 \%)$

$12(4 \%)$

$83(25 \%)$

$45(14 \%)$

$0.70(0.70,0.74)$

$240(72 \%)$

$8(2 \%)$

$38(11 \%)$

$31(9 \%)$

$18(5 \%)$

$13(4 \%)$

$11(3 \%)$

$11(3 \%)$

$6(2 \%)$

Table 4. For Study 3, demographic characteristics of the 665 participants at baseline. Continuous variables are reported as medians with 25th and 75th percentiles. Binary variables are reported as counts and percentages. Participants could indicate multiple races. "County liberalism": in the participant's county, the proportion of votes from the 2000-2016 United States presidential elections that went to the Democratic candidate. 


\begin{tabular}{|c|c|c|c|c|}
\hline Outcome & Raw mean difference & Standardized mean difference & p-value & Bonferroni p-value \\
\hline \multicolumn{5}{|l|}{ Primary outcome } \\
\hline Total meat and animal products & $-2.46[-8.78,3.85]$ & $-0.09[-0.32,0.14]$ & 0.43 & \\
\hline $\begin{array}{l}\text { Total meat and animal products } \\
\text { (target demographic) }\end{array}$ & $-1.72[-8.84,5.41]$ & $-0.07[-0.34,0.21]$ & 0.63 & \\
\hline \multicolumn{5}{|l|}{ Secondary food outcomes } \\
\hline Meat & $-0.97[-4.43,2.49]$ & $-0.07[-0.30,0.16]$ & 0.57 & 1 \\
\hline Non-meat animal products & $-1.49[-6.09,3.12]$ & $-0.07[-0.28,0.14]$ & 0.52 & 1 \\
\hline Chicken & $-0.41[-2.49,1.67]$ & $-0.04[-0.27,0.18]$ & 0.69 & 1 \\
\hline Turkey & $0.02[-0.5,0.54]$ & $0.01[-0.2,0.21]$ & 0.94 & 1 \\
\hline Fish & $0.05[-1.05,1.15]$ & $0.01[-0.18,0.20]$ & 0.93 & 1 \\
\hline Pork & $-0.28[-0.87,0.3]$ & $-0.08[-0.26,0.09]$ & 0.34 & 1 \\
\hline Beef & $-0.11[-1.05,0.83]$ & $-0.02[-0.23,0.18]$ & 0.81 & 1 \\
\hline Other meat & $-0.25[-0.62,0.12]$ & $-0.12[-0.29,0.06]$ & 0.19 & 1 \\
\hline Dairy & $-1.24[-5.29,2.8]$ & $-0.06[-0.26,0.14]$ & 0.54 & 1 \\
\hline Eggs & $-0.23[-1.76,1.29]$ & $-0.03[-0.25,0.18]$ & 0.76 & 1 \\
\hline Healthy plant foods & $5.23[-8.3,18.76]$ & $0.09[-0.14,0.32]$ & 0.44 & 1 \\
\hline \multicolumn{5}{|l|}{ Exploratory attitude outcomes } \\
\hline Importance of health & $0.00[-0.23,0.23]$ & $0.00[-0.22,0.21]$ & 0.99 & 1 \\
\hline Importance of environment & $0.00[-0.26,0.27]$ & $0.00[-0.20,0.20]$ & 0.97 & 1 \\
\hline Importance of animal welfare & $0.13[-0.26,0.52]$ & $0.10[-0.20,0.39]$ & 0.49 & 1 \\
\hline Interest in activism & & $-0.05[-0.4,0.31]$ & 0.78 & 1 \\
\hline Speciesism & & $0.08[-0.26,0.42]$ & 0.62 & 1 \\
\hline Social dominance orientation & & $0.02[-0.28,0.32]$ & 0.79 & 1 \\
\hline
\end{tabular}

Table 5. For Study 2, estimated intervention effects for the primary outcomes, secondary food outcomes, and exploratory attitude outcomes. Negative estimates represent intervention effects in the desired direction (reduced consumption). "Target demographic": Participants who reported being Democrats and having graduated 2-year college. Raw mean differences represent ounces consumed over the past week for the primary outcome and secondary food outcomes; they represent units on a 7-point Likert scale for the perceived importance items; and they are omitted for the three composite scales, which were already standardized. Brackets are $95 \%$ confidence intervals without correction for multiple testing. 


\begin{tabular}{lccc}
\hline Food & "Reduce" pledge (\%) & "Eliminate" pledge (\%) & Either pledge (\%) \\
\hline Chicken & 40 & 14 & 53 \\
Fish & 39 & 7 & 46 \\
Pork & 31 & 28 & 58 \\
Beef & 35 & 23 & 57 \\
Other meat & 36 & 25 & 60 \\
Dairy & 36 & 8 & 44 \\
Eggs & 39 & 6 & 45 \\
\hline
\end{tabular}

Table 6. For Study 3, the percent of intervention-group participants ( $n=333)$ who pledged to reduce consumption, who pledged to eliminate consumption, and who made either pledge for each food type. 


\title{
Supplement: Effectiveness of a theory-informed documentary to reduce consumption of meat and animal products
}

\author{
Contents
}

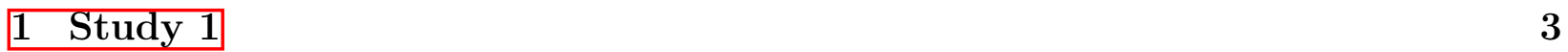

$1.1 T_{0}$ questionnaire items . . . . . . . . . . . . . . . . . 3

1.2 Demographic measures . . . . . . . . . . . . . . . . . . 3

1.2 .1 Attention check . . . . . . . . . . . . . . . . 4

$1.3 \quad T_{1}$ questionnaire items $\ldots \ldots \ldots \ldots \ldots$

1.3 .1 Food consumption . . . . . . . . . . . . . . . . 5

1.3 .2 Awareness probe . . . . . . . . . . . . . . . . . 6

1.3 .3 COVID pandemic effects on dietary agency . . . . . . . . . . . . . . . 7

1.3 .4 Exploratory attitude outcomes . . . . . . . . . . . . . . . . . . 7

1.4 Details of primary statistical analyses . . . . . . . . . . . . . . . . . 10

1.5 Additional results of sensitivity analyses . . . . . . . . . . . . . . . . 10

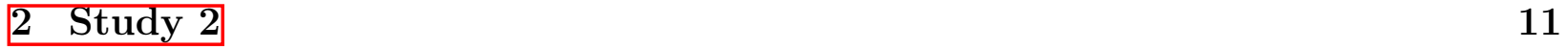

2.1 Additional results . . . . . . . . . . . . . . . . . . . . . . . . . . 11

\begin{tabular}{|lll}
\hline 3 & Study 3 & 14
\end{tabular}

3.1 Additional information on recruitment . . . . . . . . . . . . . . . . 14

$3.2 \quad T_{0}$ intervention engagement items . . . . . . . . . . . . . . . . 15

3.3 Interrim engagement email . . . . . . . . . . . . . . . . . . . 17 
3.4 Additional results of sensitivity analyses f . . . . . . . . . . . . . . . 17 


\section{STUDY 1}

\section{1. $T_{0}$ questionnaire items}

The questionnaire items are listed by chronological appearance in the questionnaire.

\subsection{Demographic measures}

Your sex:

Female

Male

Other

Your age (years):

Your highest educational level:

Did not graduate high school

Graduated high school

Graduated 2-year college

Graduated 4-year college

Obtained post-graduate degree (e.g., MS, MD, MBA, PhD, etc.)

Your ethnicity (check all that apply):
Caucasian
Hispanic
Black/African American
$\square$ Middle Eastern
$\square$ Pacific Islander 
$\square$ Native American

$\square$ South Asian

$\square$ East Asian

Southeast Asian

Your current location of residence:

[drop-down menus with states and counties]

Your political party affiliation:

Democrat

Republican

Independent

Other/I don't know

For each participant, we used state and county data, along with an external database (MIT Election Data and Science Lab, 2018), to calculate "county liberalism", defined as the proportion of votes in all 2000-2016 United States presidential elections that went to the Democratic candidate among all votes in the county that were cast for a major-party candidate.

\subsubsection{Attention check}

Which of the following points did the video make? Please select all that apply.

Regularly eating seafood is good for you.

Most Americans get less than the recommended amount of exercise.

The ways we raise animals for human consumption causes the animals to suffer.

It is important to teach children good habits in terms of exercise.

Exercising for even 10 minutes a day can improve health. 


\section{3. $T_{1}$ questionnaire items}

\subsubsection{Food consumption}

As follows, we modified the freely available National Cancer Institute Dietary Health Questionnaire III, specifically the 1-month version with portion sizes (Diet History Questionnaire III (DHQ III), 2018; Thompson et al., 2002). Our modifications were: (1) adapting the frequency options for our briefer follow-up time frame; (2) condensing individual foods into fewer categories, informed by those used in Macdonald et al. (2016); and (3) omitting a large number of foods not relevant to the present research.

We examined 15 total foods, presented in this order:

- Healthy plant-based foods (5): leafy green vegetables, other vegetables, fruits, whole grains, legumes (including beans)

- Meats (6): chicken, turkey, fish, pork, beef, other meat

- Other animal products (2): dairy, eggs

- Decoy foods (nutritionally undesirable plant-based foods) (2): refined grains (e.g., white bread), sweetened beverages such as soft drinks

For each food, we asked the following questions:

Over the past week, how often did you eat [FOOD]?

$\bigcirc$ Never

1 time in the past week

2 times in the past week

3-4 times in the past week

5-6 times in the past week

1 time per day

2 or more times per day

Each time you ate [FOOD], how much did you usually eat? 
Less than 2 ounces or less than $1 / 2$ cup

2 to 5 ounces or $1 / 2$ to 1 cup

More than 5 ounces or more than 1 cup

For analysis purposes, we expressed consumption amounts in terms of ounces per week using midpoints of each category (e.g., 3.5 for "3-4 times in the past week"), with the exception of the upper categories for frequency and amount. For these, " 2 or more times per day" was coded as 2 , and "more than 5 ounces or 1 cup" was coded as 5 .

\subsubsection{Awareness probe}

We assessed participants' potential awareness of the study's purpose using 2 multiple-choice items that mimicked a funnel debriefing. The first item asked, "What do you think the researchers doing this study were most interested in measuring?", with 10 possible responses consisting of different macronutrients and food categories. The second item asked participants why they thought the researchers measured the chosen item: to look at general consumption patterns, to increase consumption of this item, or to decrease consumption. We coded those participants who responded correctly to both items (i.e., "meat and animal product intake" followed by "to try to decrease people's meat and animal product intake") as "potentially aware".

What do you think the researchers doing this study were most interested in measuring?

Total caloric intake

Protein intake

Fat intake

Vegetable intake

Fruit intake

Whole-grain intake

Meat and animal product intake

Refined-grain intake

Sweetened beverage intake

I don't know what the researchers were interested in measuring 
For participants who did not answer "I don't know", the below questions were presented with the participant's chosen response above replacing "[CHOSEN ITEM]":

Why do you think this study measured [CHOSEN ITEM] intake?

To look at general consumption patterns and see how they vary across different demographic groups

To try to increase people's [CHOSEN ITEM] intake

To try to decrease people's [CHOSEN ITEM] intake

I don't know why the study measured this

\subsubsection{COVID pandemic effects on dietary agency}

In the past week, how much has the coronavirus pandemic affected your ability to choose what you eat?

$\begin{array}{ccccccc}1 & 2 & 3 & 4 & 5 & 6 & 7 \\ \begin{array}{c}\text { Much less } \\ \text { choice }\end{array} & \begin{array}{c}\text { Somewhat } \\ \text { less choice }\end{array} & \begin{array}{c}\text { Slightly } \\ \text { less choice }\end{array} & \text { No change } & \begin{array}{c}\text { Slightly } \\ \text { more choice }\end{array} & \begin{array}{c}\text { Somewhat } \\ \text { more choice }\end{array} & \begin{array}{c}\text { Much } \\ \text { more choice }\end{array}\end{array}$

\subsubsection{Exploratory attitude outcomes}

We collected exploratory measures of participants' attitudes and values regarding the 3 harms of animal product consumption highlighted in the documentary (health, the environment, and animal welfare) by asking participants to rate on a 7 -point scale how important they perceived each issue to be (Hekler et al., 2010). We also probed in more detail participants' attitudes regarding animal welfare because this type of educational appeal has appeared only relatively recently in dietary interventions (Mathur et al., 2021) and has received less academic study than appeals regarding health or the environment (Bianchi et al., 2018; Grundy et al., 2021). To this end, we assessed participants' interest in animal welfare activism and reform (Corning \& Myers, 2002), and we collected a measure of "speciesism", which is the assignment of a different moral standing based on species membership itself, holding constant ethically relevant traits such as sentience and the capacity to suffer (Caviola et al. 2019, Singer, 1995). Finally, we assessed participants' social dominance orientation, which is a generalized preference for group-based hierarchy and inequality that strongly predicts prejudice against a number of human social groups (e.g., poor people and ethnic minorities) and of support for policies that uphold social hierarchies (Ho et al., 2015). We collected this measure because both speciesism (Caviola et al. 2019) and meat-eating habits (Allen 
et al. 2000) are cross-sectionally associated with social dominance orientation, leading us to speculate that intervening on attitudes toward animals might reduce social dominance orientation. The activism, speciesism, and social dominance scales were standardized sums of multiple items.

Perceived importance of health, the environment, and animal welfare We used items minimally adapted from Hekler et al. (2010). We treated these three items as separate outcomes.

For each of the issues below, think about how important the issue is to you, compared to other things in your life. Show how important the issue is to you on the scale below.

$\begin{array}{llllll}1 & 2 & 3 & 4 & 5 & 6 \\ \begin{array}{l}\text { Not at all } \\ \text { important }\end{array} & \text { tant } & \text { tampor- } & \text { About as im- } \\ \text { compared to } & & \text { portant } & \text { tant } & \begin{array}{l}\text { More impor- } \\ \text { the most im- } \\ \text { portant }\end{array} & \text { tant impor- }\end{array}$

other things

in your life

1. Eating a healthful diet

2. Environmental sustainability

3. Animal welfare

Speciesism We used without modification the scale developed and validated by Caviola et al. (2019). Participants rated each item on a 7-point scale from "strongly disagree" to "strongly agree".

1. Morally, animals always count for less than humans.

2. Humans have the right to use animals however they want to.

3. It is morally acceptable to keep animals in circuses for human entertainment.

4. It is morally acceptable to trade animals like possessions.

5. Chimpanzees should have basic legal rights such as a right to life or a prohibition of torture. (reverse-coded)

6. It is morally acceptable to perform medical experiments on animals that we would not perform on any human. 
Interest in animal welfare activism and reform The first two items are modified from those with the largest eigenvalues from the 35-item Activism Orientation Scale Corning \& Myers, 2002); we modified them to pertain specifically to animal advocacy. We created the remaining three items to relate to concrete actions relevant to animal advocacy. participants rated each item on a 7-point Likert scale ranging from "extremely unlikely" to "extremely likely" (Corning \& Myers, 2002).

How likely is it that you will engage in this activity in the future?

1. Display a poster or bumper sticker with a message about animal welfare issues, such as factory farming?

2. Invite a friend to attend a meeting of an organization or event focused on animal welfare issues, such as factory farming?

3. Vote in favor of legislation that would improve animal welfare conditions on factory farms?

4. Sign a petition to a major grocery store asking them to stop selling products produced in factory farms?

5. Donate to an organization or event focused on animal welfare issues, such as factory farming?

Social dominance orientation We used without modification Ho et al. (2015)'s validated 8-item $\mathrm{SDO}_{7(s)}$ scale.

Show how much you favor or oppose each idea below by selecting a number from 1 to 7 on the scale below. You can work quickly; your first feeling is generally best.

$\begin{array}{ccccccc}1 & 2 & 3 & 4 & 5 & 6 & 7 \\ \begin{array}{c}\text { Strongly } \\ \text { oppose }\end{array} & \begin{array}{c}\text { Somewhat } \\ \text { oppose }\end{array} & \begin{array}{c}\text { Slightly } \\ \text { oppose }\end{array} & \text { Neutral } & \begin{array}{c}\text { Slightly } \\ \text { favor }\end{array} & \begin{array}{c}\text { Somewhat } \\ \text { favor }\end{array} & \begin{array}{c}\text { Strongly } \\ \text { favor }\end{array}\end{array}$

1. An ideal society requires some groups to be on top and others to be on the bottom.

2. Some groups of people are simply inferior to other groups.

3. No one group should dominate in society.

4. Groups at the bottom are just as deserving as groups at the top.

5. Group equality should not be our primary goal.

6. It is unjust to try to make groups equal. 
7. We should do what we can to equalize conditions for different groups.

8. We should work to give all groups an equal chance to succeed.

\subsection{Details of primary statistical analyses}

Multiple imputation We used fully conditional multiple imputation for all variables with missing data, including the outcome, using the $\mathrm{R}$ package mice (Buuren \& GroothuisOudshoorn, 2010; Van Buuren, 2018). We created 10 imputations (Graham et al., 2007), pooled variances across imputations using Rubin's Rules, and conducted hypothesis testing on the pooled estimates using the $t$-distribution (Dong \& Peng (2013)'s Equations (8-9)).

Calculation of standardized mean differences We calculated standardized mean differences using the Hedges' $g$ metric (Hedges, 1981), which represent intervention effects in terms of the conditional standard deviation of the outcome, $\sigma_{Y \mid X}$, where $Y$ is the outcome and $X$ is the intervention indicator. When calculating standardized mean differences for effect modifiers, we again simply standardized by $\sigma_{Y \mid X}$. We could have standardized by the estimated residual standard deviation in the multivariable regression model, which would additionally have conditioned on the effect modifiers and hence yielded larger standardized mean differences. We used $\sigma_{Y \mid X}$ for comparability with the standardized mean differences of the primary analyses.

\subsection{Additional results of sensitivity analyses}

Table S1 shows complete-case estimates of the intervention's effects on the primary outcome and all additional outcomes. 


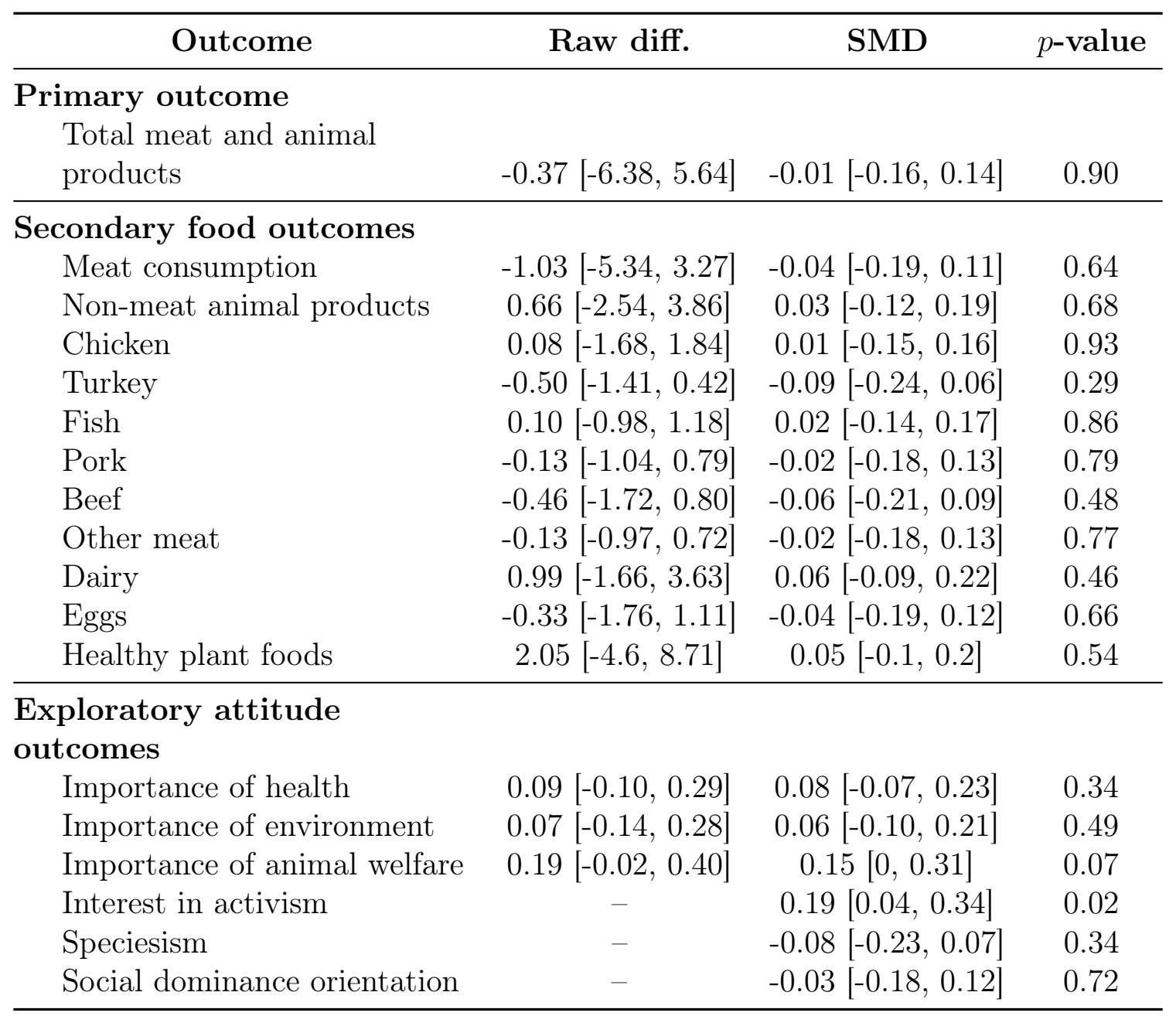

Table S1: For Study 1, complete-case estimates of intervention effects for the primary outcome, secondary food outcomes, and exploratory attitude outcomes. Negative estimates represent intervention effects in the desired direction (reduced consumption). Raw mean differences ("raw diff.") represent ounces consumed over the past week for the primary outcome and secondary food outcomes; they represent units on a 7-point Likert scale for the perceived importance items; and they are omitted for the three composite scales, which were already standardized. SMD: standardized mean difference. Brackets are $95 \%$ confidence intervals without correcting for multiple testing. p-values are not corrected for multiple testing.

\section{STUDY 2}

\subsection{Additional results}

Table S2 show participant demographics, Table S3 shows moderation estimates, and Table S4 shows estimated intervention effects on past-week food outcomes. 


\begin{tabular}{|c|c|c|}
\hline Characteristic & $\begin{array}{l}\text { Intervention } \\
\quad(\mathrm{n}=148)\end{array}$ & $\begin{array}{l}\text { Control } \\
(\mathrm{n}=152)\end{array}$ \\
\hline \multicolumn{3}{|l|}{ Sex } \\
\hline Male & $80(54 \%)$ & $83(55 \%)$ \\
\hline Female & $67(45 \%)$ & $68(45 \%)$ \\
\hline Other & $1(1 \%)$ & $1(1 \%)$ \\
\hline Age (years) & $33(26,41)$ & $32(27,42)$ \\
\hline \multicolumn{3}{|l|}{ Education } \\
\hline Did not graduate high school & $1(1 \%)$ & $2(1 \%)$ \\
\hline Graduated high school & $34(23 \%)$ & $42(28 \%)$ \\
\hline Graduated 2-year college & $23(16 \%)$ & $13(9 \%)$ \\
\hline Graduated 4-year college & $56(38 \%)$ & $55(36 \%)$ \\
\hline Completed post-graduate degree & $34(23 \%)$ & $40(26 \%)$ \\
\hline \multicolumn{3}{|l|}{ Political party } \\
\hline Democrat & $83(56 \%)$ & $86(57 \%)$ \\
\hline Independent & $28(19 \%)$ & $30(20 \%)$ \\
\hline Republican & $28(19 \%)$ & $29(19 \%)$ \\
\hline Other/I don't know & $9(6 \%)$ & $7(5 \%)$ \\
\hline County liberalism & $0.57(0.44,0.70)$ & $0.55(0.45,0.70)$ \\
\hline \multicolumn{3}{|l|}{ Race } \\
\hline Causasian & $108(73 \%)$ & $115(76 \%)$ \\
\hline Black/African American & $14(9 \%)$ & $24(16 \%)$ \\
\hline Hispanic & $11(7 \%)$ & $6(4 \%)$ \\
\hline East Asian & $9(6 \%)$ & $5(3 \%)$ \\
\hline Southeast Asian & $6(4 \%)$ & $5(3 \%)$ \\
\hline South Asian & $3(2 \%)$ & $3(2 \%)$ \\
\hline Native American & $5(3 \%)$ & $3(2 \%)$ \\
\hline Middle Eastern & $3(2 \%)$ & $2(1 \%)$ \\
\hline
\end{tabular}

Table S2: For Study 2, demographic characteristics of the 300 participants at baseline. Continuous variables are reported as medians with $25^{\text {th }}$ and $75^{\text {th }}$ percentiles. Binary variables are reported as counts and percentages. Participants could indicate multiple races. "County liberalism": in the participant's county, the proportion of votes from the 2000-2016 United States presidential elections that went to the Democratic candidate. 


\begin{tabular}{lccc}
\hline \multicolumn{1}{c}{ Coefficient } & Raw diff. & SMD & $p$-value \\
\hline Main effects & $0.45[-0.16,1.06]$ & $0.38[-0.14,0.9]$ & 0.15 \\
$\quad$ Intercept & $0.42[-0.98,1.83]$ & $0.36[-0.83,1.55]$ & 0.55 \\
Intervention (vs. control) & $0.18[-0.08,0.44]$ & $0.15[-0.07,0.37]$ & 0.17 \\
Female & $0.06[-0.26,0.38]$ & $0.05[-0.22,0.32]$ & 0.70 \\
Age $\leq 25$ years & $0.11[-0.17,0.38]$ & $0.09[-0.14,0.33]$ & 0.45 \\
At least 2-year college & $-0.10[-0.45,0.25]$ & $-0.09[-0.39,0.21]$ & 0.58 \\
Caucasian & & & \\
Independent/other & $-0.12[-0.52,0.28]$ & $-0.10[-0.44,0.24]$ & 0.56 \\
$\quad$ (vs. Republican) & $-0.29[-0.63,0.06]$ & $-0.24[-0.54,0.05]$ & 0.10 \\
Democrat & $-0.02[-0.11,0.06]$ & $-0.02[-0.09,0.06]$ & 0.63 \\
$\quad$ (vs. Republican) & & & \\
County liberalism & $0.08[-0.44,0.60]$ & $0.07[-0.37,0.51]$ & 0.76 \\
\hline Moderation of intervention & $-0.08[-0.63,0.48]$ & $-0.06[-0.54,0.41]$ & 0.79 \\
Female & $-0.07[-0.65,0.51]$ & $-0.06[-0.55,0.43]$ & 0.81 \\
Age $\leq 25$ years & $-0.07[-0.64,0.5]$ & $-0.06[-0.55,0.42]$ & 0.80 \\
At least 2-year college & & & \\
Caucasian & $0.16[-0.57,0.88]$ & $0.13[-0.48,0.75]$ & 0.67 \\
Independent/other & $0.17[-0.53,0.86]$ & $0.14[-0.45,0.73]$ & 0.64 \\
$\quad$ (vs. Republican) & $0.05[-0.11,0.21]$ & $0.04[-0.1,0.18]$ & 0.56 \\
Democrat & &
\end{tabular}

Table S3: In Study 2, estimated moderation by baseline demographic variables of intervention effects on the primary outcome (the continuous measure of intentions to reduce consumption). Positive estimates represent intervention effects in the desired direction (increased intentions to reduce consumption). Raw mean differences represent ounces consumed over the past week. Main effects represent differences in average consumption by the demographic variables. SMD: Standardized mean difference (Hedges' $g$ ). Moderation estimates represent differences in intervention effectiveness for each demographic variable, with negative values representing greater effectiveness (i.e., greater reductions in consumption). Brackets are $95 \%$ confidence intervals that do not correct for multiple testing. "County liberalism" represents a 10-percentage point higher proportion of votes cast for Democratic presidential candidates in the participant's county. 


\begin{tabular}{llll}
\hline \multicolumn{1}{c}{ Outcome } & \multicolumn{1}{c}{ Raw diff. } & \multicolumn{1}{c}{ SMD } & $p$-value \\
\hline Past food outcomes & & & \\
Total meat and animal products & $-4.07[-11.74,3.60]$ & $-0.12[-0.35,0.11]$ & 0.30 \\
Meat consumption & $-3.57[-8.76,1.63]$ & $-0.15[-0.38,0.07]$ & 0.18 \\
Non-meat animal products & $-0.50[-4.94,3.93]$ & $-0.03[-0.25,0.20]$ & 0.82 \\
Chicken & $-2.14[-3.93,-0.35]$ & $-0.27[-0.50,-0.04]$ & 0.02 \\
Turkey & $-1.08[-2.23,0.07]$ & $-0.21[-0.44,0.02]$ & 0.07 \\
Fish & $-0.12[-2.19,1.96]$ & $-0.01[-0.24,0.21]$ & 0.91 \\
Pork & $0.41[-0.56,1.39]$ & $0.10[-0.13,0.32]$ & 0.41 \\
Beef & $-0.47[-1.89,0.95]$ & $-0.08[-0.30,0.15]$ & 0.52 \\
Other meat & $-0.17[-1.18,0.84]$ & $-0.04[-0.27,0.19]$ & 0.74 \\
Dairy & $0.77[-2.85,4.38]$ & $0.05[-0.18,0.28]$ & 0.68 \\
Eggs & $-1.27[-3.47,0.93]$ & $-0.13[-0.36,0.10]$ & 0.26 \\
Healthy plant foods & $10.87[-0.41,22.15]$ & $0.22[-0.01,0.45]$ & 0.06 \\
\hline Exploratory attitude outcomes & & & \\
Importance of health & $-0.01[-0.28,0.26]$ & $-0.01[-0.23,0.22]$ & 0.96 \\
Importance of environment & $0.30[-0.01,0.61]$ & $0.22[-0.01,0.45]$ & 0.05 \\
Importance of animal welfare & $0.22[-0.07,0.51]$ & $0.17[-0.05,0.40]$ & 0.13 \\
Interest in activism & - & $0.16[-0.07,0.39]$ & 0.17 \\
Speciesism & - & $-0.05[-0.28,0.18]$ & 0.66 \\
Social dominance orientation & - & $-0.02[-0.25,0.21]$ & 0.86 \\
\hline
\end{tabular}

Table S4: In Study 2, estimated intervention effects on secondary outcomes. Raw mean differences represent ounces consumed over the past week for the primary outcome and secondary food outcomes; they represent units on a 7-point Likert scale for the perceived importance items; and they are omitted for the three composite scales, which were already standardized. SMD: Standardized mean difference (Hedges'g). Brackets are 95\% confidence intervals that do not correct for multiple testing.

\section{STUDY 3}

\subsection{Additional information on recruitment}

The registry from which we recruited participants was built over 20 years and approximately 20 human nutrition intervention trials run by the Gardner Nutrition Studies Research Group at Stanford University. The registry includes participants who at at some point had completed an online screener to be a potential participant in at least one of the past trials, and agreed to allow their contact information to be kept for the purpose of being informed about future studies. Approximately 25\% of individuals in the registry are past study participants. The remaining individuals either had not been eligible for the particular study for which they were screened, or who had been eligible but ultimately chose not to participate after learning 
about the details of participation. With every new announcement to the registry of new research participation opportunities, those receiving the notification are informed that they can be removed from the registry simply be replying to the notification with this request.

\section{2. $T_{0}$ intervention engagement items}

For participants in the intervention group only, the documentary was presented as 3 sequential chunks (corresponding to content about health, the environment, and animal welfare), each followed by questions about its content as follows.

After the health component of the documentary:

As discussed in the video, plant-based diets can have several health benefits. Can you name at least 2 of these benefits that you found compelling?

[Free-text box]

After the environment component of the documentary:

As discussed in the video, the production of meat and animal products causes several types of environmental damage. Can you name at least 2 of these damages that you found compelling?

[Free-text box]

After the animal welfare component of the documentary:

As discussed in the video, the production of meat and animal products causes significant suffering to animals in many ways. Can you name at least 2 ways in which farmed animals suffer that you found compelling?

[Free-text box]

For participants assigned to view the documentary, we asked following questions after participants viewed the documentary.

Many of our previous Stanford research participants have pledged to eat and drink less meat and animal products after watching this documentary. Are you willing to take one of the following pledges?

[Matrix of radio buttons with foods listed as rows (chicken, fish, pork, beef, all other meats, eggs, dairy) and pledges listed as columns ("Yes, I pledge to stop eating this food", "Yes, I pledge to eat this food less often", "No, I do not wish to make a pledge about this food")] 
Participants who chose one of the pledges then completed the following items.

Congratulations on making your pledge.

Research shows that making concrete goals helps us to follow through on our intentions to change our behavior. A good goal is specific, measurable, and attainable, and has a deadline.

For example, "I will stop eating all meat, eggs, and dairy by XXX date". Please pick a date on which you plan to meet your pledge goal.

I will meet my pledge goal by the following date: [Clickable calendar]

Research also shows that how we eat is largely habitual. It can be initially hard to break out of old habits, but once we form new habits, they can become just as automatic and easy to maintain as the old habits they replace.

What specific strategies will you try in order to form a habit around your new goals? Please check as many as you would like. These are just examples - use your imagination!

On the same day every week, I will prepare several servings of plant-based meals so that I don't have to think about it during the weekday.

For a specific meal (e.g., weekday lunches), I will always buy take-out from restaurants that have plant-based options.

I will meet my pledge goal a gradual way by eliminating one meat or animal product at a time (e.g., first chicken, then eggs, etc.).

I will choose a specific day of the week (e.g., Monday) when I will eat only plantbased meals.

I will choose a specific meal (e.g., dinner) when I will eat only plant-based meals.

Other (please specify):

Last, one of the most effective ways to form new dietary habits is to keep a diary to track what you are eating. Our participants tell us this has been a very helpful way to meet their pledge goals.

For example, you could have a calendar and check off each day you were able to meet your pledge goal - try to make the longest streak you can! There are also smartphone apps for this purpose (e.g., Streaks, Habit List, Chains.cc, and many more). 


\subsection{Interrim engagement email}

One week after random assignment, we again emailed pledge-makers. The email was titled "Remember your meat pledge last week?", and its content was:

A week ago, you watched a documentary about how meat and animal products are produced, and you joined many of our Stanford participants in making a pledge to eat less meat, eggs, and/or dairy.

You pledged to stop eating the following foods: [Piped text from matrix question]

You pledged to eat less of the following foods: [Piped text from matrix question]

You planned to meet your pledge goals by this following date: [Piped text from date question]

And you planned to try the following strategies to meet your pledge goals: [Piped text from strategies question]

Keep it up! It takes about 2 weeks for new habits to start to feel easy, so you are already halfway there.

\subsection{Additional results of sensitivity analyses}

Table S5 shows complete-case estimates of the intervention's effects on the primary outcome and all additional outcomes. 


\begin{tabular}{|c|c|c|c|}
\hline Outcome & Raw diff. & SMD & $p$-value \\
\hline \multicolumn{4}{|l|}{ Primary outcomes } \\
\hline $\begin{array}{l}\text { Total meat and animal } \\
\text { products }\end{array}$ & $-5.37[-13.33,2.59]$ & $-0.18[-0.44,0.09]$ & 0.19 \\
\hline $\begin{array}{l}\text { Total meat and animal } \\
\text { products (target demographic) }\end{array}$ & $-3.66[-12.89,5.56]$ & $-0.14[-0.48,0.21]$ & 0.43 \\
\hline \multicolumn{4}{|l|}{ Secondary food outcomes } \\
\hline Meat consumption & $-2.34[-6.67,1.99]$ & $-0.14[-0.41,0.12]$ & 0.29 \\
\hline Non-meat animal products & $-3.03[-8.89,2.82]$ & $-0.14[-0.4,0.13]$ & 0.31 \\
\hline Chicken & $-1.58[-3.71,0.55]$ & $-0.19[-0.46,0.07]$ & 0.14 \\
\hline Turkey & $0.04[-0.61,0.7]$ & $0.02[-0.25,0.28]$ & 0.89 \\
\hline Fish & $0.74[-0.82,2.29]$ & $0.13[-0.14,0.39]$ & 0.35 \\
\hline Pork & $-0.77[-1.67,0.14]$ & $-0.22[-0.49,0.04]$ & 0.10 \\
\hline Beef & $-0.16[-1.65,1.33]$ & $-0.03[-0.29,0.24]$ & 0.84 \\
\hline Other meat & $-0.61[-1.28,0.05]$ & $-0.24[-0.5,0.02]$ & 0.07 \\
\hline Dairy & $-2.62[-7.66,2.42]$ & $-0.14[-0.4,0.13]$ & 0.31 \\
\hline Eggs & $-0.41[-2.57,1.74]$ & $-0.05[-0.32,0.21]$ & 0.71 \\
\hline Healthy plant foods & $7.45[-7.54,22.45]$ & $0.13[-0.13,0.4]$ & 0.33 \\
\hline \multicolumn{4}{|l|}{$\begin{array}{l}\text { Exploratory attitude } \\
\text { outcomes }\end{array}$} \\
\hline Importance of health & $0.00[-0.29,0.29]$ & $0.00[-0.27,0.27]$ & 0.99 \\
\hline Importance of environment & $0.01[-0.3,0.32]$ & $0.01[-0.26,0.28]$ & 0.96 \\
\hline Importance of animal welfare & $0.29[-0.05,0.64]$ & $0.23[-0.04,0.5]$ & 0.09 \\
\hline Interest in activism & - & $0.08[-0.19,0.35]$ & 0.58 \\
\hline Speciesism & - & $0.07[-0.2,0.34]$ & 0.62 \\
\hline Social dominance orientation & - & $-0.01[-0.29,0.26]$ & 0.92 \\
\hline
\end{tabular}

Table S5: In Study 3, complete-case estimates of intervention effects for the primary outcomes, secondary food outcomes, and exploratory attitude outcomes. Negative estimates represent intervention effects in the desired direction (reduced consumption). "Target demographic": Participants who reported being Democrats with at least a 2-year college education. Raw mean differences ("raw diff.") represent ounces consumed over the past week for the primary outcome and secondary food outcomes; they represent units on a 7-point Likert scale for the perceived importance items; and they are omitted for the three composite scales, which were already standardized. "SMD": standardized mean difference. Brackets are $95 \%$ confidence intervals that do not correct for multiple testing. p-values are not corrected for multiple testing. 


\section{REFERENCES}

Allen, M. W., Wilson, M., Ng, S. H., \& Dunne, M. (2000). Values and beliefs of vegetarians and omnivores. The Journal of Social Psychology, 140(4), 405-422.

Bianchi, F., Dorsel, C., Garnett, E., Aveyard, P., \& Jebb, S. A. (2018). Interventions targeting conscious determinants of human behaviour to reduce the demand for meat: A systematic review with qualitative comparative analysis. International Journal of Behavioral Nutrition and Physical Activity, 15(1), 102.

Buuren, S. v., \& Groothuis-Oudshoorn, K. (2010). mice: Multivariate imputation by chained equations in R. Journal of Statistical Software, 1-68.

Caviola, L., Everett, J. A., \& Faber, N. S. (2019). The moral standing of animals: Towards a psychology of speciesism. Journal of Personality and Social Psychology, 116(6), 1011.

Corning, A. F., \& Myers, D. J. (2002). Individual orientation toward engagement in social action. Political Psychology, 23(4), 703-729.

Diet History Questionnaire III (DHQ III) (Tech. Rep.). (2018, March). National Cancer Institute. (https://epi.grants.cancer.gov/dhq3/)

Dong, Y., \& Peng, C.-Y. J. (2013). Principled missing data methods for researchers. SpringerPlus, 2(1), 222.

Graham, J. W., Olchowski, A. E., \& Gilreath, T. D. (2007). How many imputations are really needed? some practical clarifications of multiple imputation theory. Prevention Science, 8(3), 206-213.

Grundy, E., Slattery, P., Saeri, A. K., Watkins, K., Houlden, T., Farr, N., ... others (2021). Interventions that influence animal-product consumption: A meta-review. Future Foods. (Preprint retrieved from https://osf.io/mcdsq/)

Hedges, L. V. (1981). Distribution theory for Glass's estimator of effect size and related estimators. Journal of Educational Statistics, 6(2), 107-128.

Hekler, E. B., Gardner, C. D., \& Robinson, T. N. (2010). Effects of a college course about food and society on students' eating behaviors. American Journal of Preventive Medicine, $38(5), 543-547$.

Ho, A. K., Sidanius, J., Kteily, N., Sheehy-Skeffington, J., Pratto, F., Henkel, K. E., ... Stewart, A. L. (2015). The nature of social dominance orientation: Theorizing and measuring preferences for intergroup inequality using the new $\mathrm{SDO}_{7}$ scale. Journal of Personality and Social Psychology, 109(6), 1003.

Macdonald, B., Caldwell, K., \& Boese, G. (2016). The effects of 'reduce' and 'eliminate' appeals on individual meat consumption (Tech. Rep.). Reducetarian Foundation. (https:// osf.io/nxrx3/) 
Mathur, M. B., Peacock, J., Reichling, D. B., Nadler, J., Bain, P. A., Gardner, C. D., \& Robinson, T. N. (2021). Interventions to reduce meat consumption by appealing to animal welfare: Meta-analysis and evidence-based recommendations. Appetite, 164, 105277.

MIT Election Data and Science Lab. (2018). County Presidential Election Returns 20002016. Harvard Dataverse. Retrieved from https://doi.org/10.7910/DVN/VOQCHQ doi: $10.7910 / \mathrm{DVN} / \mathrm{VOQCHQ}$

Singer, P. (1995). Animal liberation. Random House.

Thompson, F. E., Subar, A. F., Brown, C. C., Smith, A. F., Sharbaugh, C. O., Jobe, J. B., ... Ziegler, R. G. (2002). Cognitive research enhances accuracy of food frequency questionnaire reports: results of an experimental validation study. Journal of the American Dietetic Association, 102(2), 212-225.

Van Buuren, S. (2018). Flexible imputation of missing data. CRC press. 